March 12, 2013

KEK-TH-1544

\title{
Superfield description of gravitational couplings in generic 5D supergravity
}

\author{
Yutaka Sakamura* \\ KEK Theory Center, Institute of Particle and Nuclear Studies, KEK, \\ Tsukuba, Ibaraki 305-0801, Japan \\ Department of Particles and Nuclear Physics, \\ The Graduate University for Advanced Studies (Sokendai), \\ Tsukuba, Ibaraki 305-0801, Japan
}

\begin{abstract}
We complete the $\mathcal{N}=1$ superfield action for the generic system of vector multiplets and hypermultiplets coupled to $5 \mathrm{D}$ supergravity, which is based on the superconformal formulation. Especially we clarify the gravitational couplings to the bulk matters at linear order in the gravitational superfields. They consist of four $\mathcal{N}=1$ superfields, two of which are $Z_{2}$-odd when the fifth dimension is compactified on $S^{1} / Z_{2}$. This formulation provides a powerful tool to calculate quantum effects, keeping the $\mathcal{N}=1$ off-shell structure.
\end{abstract}

*e-mail address: sakamura@post.kek.jp 


\section{Introduction}

Higher dimensional supergravity (SUGRA) has been attracted much attention and extensively investigated in various aspects, such as effective theories of the superstring theory or M-theory, AdS/CFT correspondence [1], the model building in the context of the braneworld scenario, etc. Among them, five-dimensional (5D) SUGRA compactified on an orbifold $S^{1} / Z_{2}$ has been thoroughly investigated since it is shown to appear as an effective theory of the strongly coupled heterotic string theory [2] compactified on a Calabi-Yau 3fold [3]. Besides, the supersymmetric (SUSY) extensions of the Randall-Sundrum model [4] are also constructed in 5D SUGRA on $S^{1} / Z_{2}$ [5, 6, 7].

The general form of 5D SUGRA in the component field formalism has been clarified in the following papers. The couplings of vector multiplets, tensor multiplets and hypermultiplets to SUGRA are discussed in Refs. [8], [9] and [10], respectively. The results obtained in these works are reproduced in the superconformal formulation [11]-[18], which is a systematic method to construct generic off-shell action of 5D SUGRA.

Superfield formulation is an elegant and systematic way to construct SUSY theories. For five and six dimensions, the projective superspace approach offers nice off-shell formulations of SUGRA [19, 20]. On the other hand, such full superfield formulation is generically complicated and requires many auxiliary fields because of $\mathcal{N}=2 \mathrm{SUSY} 1$

It is useful and convenient to express higher dimensional SUSY theories in terms of $\mathcal{N}=1$ superfields. Such superfield formulation enables us to describe interactions between fields localized on the brane and those in the bulk in a transparent manner. It also makes it easier to derive the low-energy four-dimensional (4D) effective theory that preserves $\mathcal{N}=1$ SUSY. In the global SUSY case, such description of 5-10 dimensional SUSY theories were provided in Ref. [21]. Especially 5D SUSY theories have been extensively investigated by the $\mathcal{N}=1$ superfield description [22, 23, 24]. There are also some works along this direction for 5D SUGRA. In Ref. [25], the linearized minimal 5D SUGRA is constructed in $\mathcal{N}=1$ superspace. This formulation is useful to calculate quantum loop effects from the gravitational fields propagating in the 5D bulk. However it is unclear how to extend their formulation to more generic case in which matter fields also propagate in the bulk 2

Another $\mathcal{N}=1$ description of 5D SUGRA is based on the superconformal formulation. Since each 5D superconformal multiplet can be decomposed into $\mathcal{N}=1$ multiplets [17],

\footnotetext{
${ }^{1} \mathcal{N}=1$ denotes SUSY with four supercharges in this paper.

${ }^{2}$ We refer to fields other than the gravitational fields as matters in this paper.
} 
it is possible to express the generic 5D SUGRA action in terms of $\mathcal{N}=1$ superfields. This has been done in Ref. [26, 27], and used to derive the 4D effective theories [28]-[32]. However, the superfield action in these works is not a complete one. They did not take into account the $Z_{2}$-odd part of the gravitatioinal multiplet, and some terms involving the $Z_{2}$-even gravitational fields are also missing. These deficits are irrelevant if we focus on low-energy observables calculated at the classical level since $Z_{2}$-odd fields do not have zero-modes that appear in low-energy effective theory. However, when we discuss quantum loop effects, we have to take into account all fields in the theory, including the $Z_{2}$-odd fields. The purpose of this paper is to extend the results of Ref. [26, 27] to include all the gravitational fields, and complete the $\mathcal{N}=1$ superfield action for the generic system of vector multiplets and hypermultiplets coupled to 5D SUGRA 3 In this paper, we keep terms up to linear order in the gravitational superfields for each interaction term. This is enough for one-loop calculations. Thus our work can also be understood as an extension of Ref. 25] to a case that the matter superfields propagate in the bulk.

The paper is organized as follows. In the next two sections, we review our previous works. We provide the superfield description of 4D SUGRA based on the superconformal formulation in Sec. 2, and that of 5D SUGRA in which the gravitational fluctuation fields are dropped in Sec. 3. In Sec. 4, the gravitational superfields are introduced as the connections for the 5D superconformal symmetries. Their couplings to the matter superfields are determined from the invariance of the action. Sec. 5 is devoted to the summary. In Appendix A, we check that 4D field strength superfields defined in Sec. 2 correctly transform as superconformal chiral multiplets. In Appendix B, we collect explicit expressions of $\mathcal{N}=1$ matter superfields in terms of component fields of 5D superconformal multiplets. In Appendix C, we show the invariance of the action under the supergauge and the $Z_{2}$-odd superconformal transformations in the superfield description.

\section{$2 \quad 4 \mathrm{D}$ supergravity}

In this section, we review our previous work [33] that derives the superfield description of 4D SUGRA based on the superconformal formulation of Ref. [34]. This is a modified

\footnotetext{
${ }^{3}$ In this paper, we will not consider 5D tensor multiplets, which are discussed in Ref. 9, 18, just for simplicity. For practical purposes, our setup is useful enough because we can construct various phenomenological models without them. It is possible to incorporate the $5 \mathrm{D}$ tensor multiplets into our formulation by using the results in Ref. [18.
} 
version of the superfield formalism in Ref. 35] that makes a relation to the formalism of Ref. [34] manifest. We will use formulae in this section to express 5D SUGRA action in Sec. 4 .

We assume that the background geometry is a flat 4D Minkowski spacetime. Basically we use the two-component spinor notations of Ref. [36], except for the metric and the spinor derivatives. We take the background metric as $\eta_{\mu \nu}=\operatorname{diag}(1,-1,-1,-1)$ so as to match it to that of Ref. [34], and we define the spinor derivatives $D_{\alpha}$ and $\bar{D}_{\dot{\alpha}}$ as

$$
D_{\alpha} \equiv \frac{\partial}{\partial \theta^{\alpha}}-i\left(\sigma^{\mu} \bar{\theta}\right)_{\alpha} \partial_{\mu}, \quad \bar{D}_{\dot{\alpha}} \equiv-\frac{\partial}{\partial \bar{\theta}^{\dot{\alpha}}}+i\left(\theta \sigma^{\mu}\right)_{\dot{\alpha}} \partial_{\mu}
$$

which satisfy $\left\{D_{\alpha}, \bar{D}_{\dot{\alpha}}\right\}=2 i \sigma_{\alpha \dot{\alpha}}^{\mu} \partial_{\mu}$.

\subsection{Definition of superfields}

The 4D superconformal algebra consists of the translation $\boldsymbol{P}$, the Lorentz transformation $\boldsymbol{M}$, SUSY $\boldsymbol{Q}$, the R symmetry $U(1)_{A}$, the dilatation $\boldsymbol{D}$, the conformal SUSY $\boldsymbol{S}$ and the conformal boost $\boldsymbol{K}$. Among the gauge fields for these symmetries, only the vierbein $e_{\mu}^{\underline{\mu}}$, the gravitino $\psi_{\mu}$, the $U(1)_{A}$ gauge field $A_{\mu}$ and the $\boldsymbol{D}$ gauge field $b_{\mu}$ are independent degrees of freedom [34]. In our previous work [33], we showed that these fields form the following real superfield with an external Lorentz index.

$$
U^{\mu}=\left(\theta \sigma^{\nu} \bar{\theta}\right) \tilde{e}_{\nu}^{\mu}+i \bar{\theta}^{2}\left(\theta \sigma^{\nu} \bar{\sigma}^{\mu} \psi_{\nu}\right)-i \theta^{2}\left(\bar{\theta} \bar{\sigma}^{\nu} \sigma^{\mu} \bar{\psi}_{\nu}\right)+\frac{1}{4} \theta^{2} \bar{\theta}^{2}\left(3 A^{\mu}-\epsilon^{\mu \nu \rho \tau} \partial_{\nu} \tilde{e}_{\rho \tau}\right)
$$

where $\tilde{e}_{\nu}{ }^{\mu} \equiv e_{\nu}{ }^{\underline{\mu}}-\delta_{\nu}{ }^{\mu}$ is the fluctuation around the background 4 In our formulation, we keep the gravitational fields $\left(\tilde{e}_{\mu}{ }^{\nu}, \psi_{\mu}, A_{\mu}\right)$ up to linear order. Therefore we need not discriminate the curved indices $\mu, \nu, \cdots$ from the flat ones $\underline{\mu}, \underline{\nu}, \cdots$ since the background geometry is flat. Thus we omit the underlines of the flat indices in the following.

A (superconformal) chiral multiplet $\left[\phi, \chi_{\alpha}, F\right]$ is expressed by the following chiral superfield.

$$
\Phi=\left(1+\frac{w}{3} \mathcal{E}\right)\left(\phi+\theta \chi+\theta^{2} F\right)
$$

where $w$ is the Weyl weight (i.e., the $\boldsymbol{D}$ charge) of this multiplet 5 and

$$
\mathcal{E} \equiv \tilde{e}_{\mu}^{\mu}-2 i \theta \sigma^{\mu} \bar{\psi}_{\mu}
$$

\footnotetext{
${ }^{4}$ In the formulation of Ref. 34], the $\boldsymbol{D}$ gauge field $b_{\mu}$ does not play any essential role, and can be set to zero. This corresponds to the gauge fixing condition for $\boldsymbol{K}$.

${ }^{5}$ The Weyl weight of a multiplet denotes that of the lowest component in the multiplet.
} 
corresponds to the fluctuation part of the chiral density multiplet in Ref. [36]. We have worked in the chiral coordinate $y^{\mu} \equiv x^{\mu}-i \theta \sigma^{\mu} \bar{\theta}$ to express these chiral superfields. In the superconformal formulation of Ref. [34], there is a formula that embeds a chiral multiplet into a general multiplet. It is expressed in our superfield description as

$$
\mathcal{U}(\Phi) \equiv\left(1+i U^{\mu} \partial_{\mu}\right) \Phi, \quad \mathcal{U}(\bar{\Phi}) \equiv\left(1-i U^{\mu} \partial_{\mu}\right) \bar{\Phi}
$$

Each chiral superfield $\Phi$ in the full superspace integral $\int d^{4} \theta$ must appear in this form.

A real general multiplet $\left[C, \zeta_{\alpha}, \mathcal{H}, B_{\mu}, \lambda_{\alpha}, D\right]$ is expressed 6 by a real superfields,

$$
\begin{aligned}
V=\left\{1+\frac{w}{6}(\mathcal{E}+\overline{\mathcal{E}})\right\}\{ & C+i \theta \zeta-i \bar{\theta} \bar{\zeta}-\theta^{2} \mathcal{H}-\bar{\theta}^{2} \overline{\mathcal{H}}-\left(\theta \sigma^{\mu} \bar{\theta}\right) B_{\mu}^{\prime} \\
& \left.+i \theta^{2}\left(\bar{\theta} \bar{\lambda}^{\prime}\right)-i \bar{\theta}^{2}\left(\theta \lambda^{\prime}\right)+\frac{1}{2} \theta^{2} \bar{\theta}^{2} D^{\prime}\right\}
\end{aligned}
$$

where $w$ is the Weyl weight of this multiplet, and

$$
\begin{aligned}
B_{\mu}^{\prime} \equiv & B_{\mu}-\zeta \psi_{\mu}-\bar{\zeta} \bar{\psi}_{\mu}-\frac{w}{2} C A_{\mu} \\
\lambda_{\alpha}^{\prime} \equiv & \lambda_{\alpha}-\frac{i}{2}\left\{\sigma^{\mu}\left(e^{-1}\right)_{\mu}^{\nu} \partial_{\nu} \bar{\zeta}\right\}_{\alpha}-\left(\sigma^{\mu} \bar{\sigma}^{\nu} \psi_{\mu}\right)_{\alpha} B_{\nu}-\frac{w}{4}\left(\sigma^{\mu} \bar{\zeta}\right)_{\alpha} A_{\mu} \\
D^{\prime} \equiv & D-\frac{1}{2} g^{\mu \nu} \partial_{\mu} \partial_{\nu} C-\left(\bar{\lambda} \bar{\sigma}^{\mu} \psi_{\mu}-\frac{i}{2} \partial_{\nu} \zeta \sigma^{\mu} \bar{\sigma}^{\nu} \psi_{\mu}-i \partial_{\mu} \zeta \psi^{\mu}-\frac{2 i w}{3} \zeta \sigma^{\mu \nu} \partial_{\nu} \psi_{\mu}+\text { h.c. }\right) \\
& +\left(\frac{3-w}{2} A^{\mu}-\frac{1}{2} \epsilon^{\mu \nu \rho \tau} \partial_{\nu} \tilde{e}_{\rho \tau}\right) B_{\mu} .
\end{aligned}
$$

Here $\left(e^{-1}\right)_{\mu}{ }^{\nu} \equiv \delta_{\mu}{ }^{\nu}-\tilde{e}_{\mu}{ }^{\nu}$ and $g^{\mu \nu} \equiv \eta^{\mu \nu}-\tilde{e}^{\mu \nu}-\tilde{e}^{\nu \mu}$ are the inverse matrices of the vierbein and the metric, respectively.

The gauge multiplet is a real multiplet with $w=0$. The gauge field $\hat{B}_{\mu}$ is identified with

$$
\hat{B}_{\mu} \equiv\left(\delta_{\mu}{ }^{\nu}+\tilde{e}_{\mu}{ }^{\nu}\right) B_{\nu}^{\prime}=\left(\delta_{\mu}{ }^{\nu}+\tilde{e}_{\mu}{ }^{\nu}\right) B_{\nu}-\zeta \psi_{\mu}-\bar{\zeta} \bar{\psi}_{\mu}
$$

For simplicity, we consider a case of the Abelian gauge group. An extension to the nonAbelian case is straightforward as explained in Sec. 2.3. The (super)gauge transformation is expressed in our superfield description as

$$
V \rightarrow V+\mathcal{U}(\Lambda)+\mathcal{U}(\bar{\Lambda})
$$

where the transformation parameter $\Lambda=\phi^{\Lambda}+\theta \chi^{\Lambda}+\theta^{2} F^{\Lambda}$ (in the $y^{\mu}$-coordinate) is a chiral superfield. Note that $\Lambda$ must be embedded into a general multiplet by $\mathcal{U}$ in order to be

\footnotetext{
${ }^{6}$ A complex scalar $\mathcal{H}$ should be understood as $\frac{1}{2}(H+i K)$ in the notation of Ref. [34].
} 
added to $V$. We can move to the Wess-Zumino gauge by choosing $\Lambda$ as 7

$$
\operatorname{Re} \phi^{\Lambda}=-\frac{1}{2} C, \quad \chi_{\alpha}^{\Lambda}=-i \zeta_{\alpha}, \quad F^{\Lambda}=\mathcal{H}
$$

In this gauge, $V$ is written as

$$
\begin{aligned}
V_{\mathrm{WZ}}= & -\left(\theta \sigma^{\mu} \bar{\theta}\right)\left(e^{-1}\right)_{\mu}{ }^{\nu} \hat{B}_{\nu}+i \theta^{2} \bar{\theta}\left\{\bar{\lambda}-\left(\bar{\sigma}^{\nu} \sigma^{\mu} \bar{\psi}_{\nu}\right) \hat{B}_{\mu}\right\}-i \bar{\theta}^{2} \theta\left\{\lambda-\left(\sigma^{\nu} \bar{\sigma}^{\mu} \psi_{\nu}\right) \hat{B}_{\mu}\right\} \\
& +\frac{1}{2} \theta^{2} \bar{\theta}^{2}\left\{D-\left(\bar{\lambda} \bar{\sigma}^{\mu} \psi_{\mu}+\text { h.c. }\right)+\left(\frac{3}{2} A^{\mu}-\frac{1}{2} \epsilon^{\mu \nu \rho \tau} \partial_{\nu} \tilde{e}_{\rho \tau}\right) \hat{B}_{\mu}\right\},
\end{aligned}
$$

where $\hat{B}_{\mu}$ is understood as the gauge-transformed gauge field. A set of the components $\left[\hat{B}_{\mu}, \lambda_{\alpha}, D\right]$ form a gauge multiplet.

\subsection{Superconformal transformation}

Throughout this paper, we neglect terms beyond linear order in $U^{\mu}$ in the action, except for its kinetic terms that are discussed in Sec. 2.4.2. Hence it is enough to ensure an invariance of the action under the superconformal transformations up to the zeroth order in $U^{\mu}$ because it transforms inhomogeneously. The (linearized) superconformal transformations of the superfields defined in the previous subsection are expressed as

$$
\begin{aligned}
\delta_{\mathrm{sc}} U^{\mu} & =\frac{1}{2} \sigma_{\alpha \dot{\alpha}}^{\mu}\left(\bar{D}^{\dot{\alpha}} L^{\alpha}-D^{\alpha} \bar{L}^{\dot{\alpha}}\right), \\
\delta_{\mathrm{sc}} \Phi & =\left\{-\frac{1}{4} \bar{D}^{2} L^{\alpha} D_{\alpha}-i \sigma_{\alpha \dot{\alpha}}^{\mu} \bar{D}^{\dot{\alpha}} L^{\alpha} \partial_{\mu}-\frac{w}{12} \bar{D}^{2} D^{\alpha} L_{\alpha}\right\} \Phi, \\
\delta_{\mathrm{sc}} V & =\left\{-\frac{1}{4} \bar{D}^{2} L^{\alpha} D_{\alpha}-\frac{i}{2} \sigma_{\alpha \dot{\alpha}}^{\mu} \bar{D}^{\dot{\alpha}} L^{\alpha} \partial_{\mu}-\frac{w}{24} \bar{D}^{2} D^{\alpha} L_{\alpha}+\text { h.c. }\right\} V,
\end{aligned}
$$

where $L_{\alpha}$ is a transformation parameter superfield 8 Define

$$
\begin{aligned}
\xi^{\mu} & \equiv-\left.\operatorname{Re}\left(i \sigma_{\alpha \dot{\alpha}}^{\mu} \bar{D}^{\dot{\alpha}} L^{\alpha}\right)\right|_{0}, \quad \epsilon_{\alpha} \equiv-\left.\frac{1}{4} \bar{D}^{2} L_{\alpha}\right|_{0}, \\
\lambda_{\mu \nu} & \equiv-\left.\frac{1}{2} \operatorname{Re}\left\{\left(\sigma_{\mu \nu}\right)_{\beta}^{\alpha} D_{\alpha} \bar{D}^{2} L^{\beta}\right\}\right|_{0},\left.\quad \varphi_{D} \equiv \operatorname{Re}\left(\frac{1}{4} D^{\alpha} \bar{D}^{2} L_{\alpha}\right)\right|_{0}, \\
\vartheta_{A} & \left.\equiv \operatorname{Im}\left(-\frac{1}{6} D^{\alpha} \bar{D}^{2} L_{\alpha}\right)\right|_{0}, \quad \eta_{\alpha} \equiv-\left.\frac{1}{32} D^{2} \bar{D}^{2} L_{\alpha}\right|_{0},
\end{aligned}
$$

where the symbol $\left.\right|_{0}$ denotes the lowest component of a superfield. Then these components are identified with the transformation parameters for $\boldsymbol{P}, \boldsymbol{Q}, \boldsymbol{M}, \boldsymbol{D}, U(1)_{A}$ and $\boldsymbol{S}$, respectively. We have explicitly checked that (2.12) reproduces the correct superconformal transformations of each component field listed in Ref. [34].

\footnotetext{
7 This gauge is possible only in the case of $w=0$.

${ }^{8}$ We have set $\Omega^{\mu}$ in Ref. 33] to zero. This is always possible by imposing constraints on the components of $L_{\alpha}$.
} 


\section{$2.3 \quad$ Field strength superfield}

In the Abelian case, we define

$$
X \equiv\left(1+\frac{1}{4} U^{\mu} \bar{\sigma}_{\mu}^{\dot{\alpha} \alpha}\left[D_{\alpha}, \bar{D}_{\dot{\alpha}}\right]\right) V .
$$

Then its gauge transformation becomes simpler,

$$
X \rightarrow X+\Lambda+\bar{\Lambda} .
$$

Hence, a naive definition of a field strength superfield,

$$
\mathcal{W}_{\alpha}^{\text {naive }}=-\frac{1}{4} \bar{D}^{2} D_{\alpha} X
$$

is gauge-invariant. However this does not transform correctly under the superconformal transformation. From (2.12), we see that $\delta_{\mathrm{sc}} \mathcal{W}_{\alpha}^{\text {naive }}$ contains $\bar{L}_{\dot{\alpha}}$, which must be absent in the transformation of a chiral superfield. Thus we modify (2.16) as

$$
\mathcal{W}_{\alpha}=-\frac{1}{4} \bar{D}^{2}\left[D_{\alpha} X\right]_{\mathbb{E}}
$$

where

$$
\left[D_{\alpha} X\right]_{\mathbb{E}} \equiv D_{\alpha} X-\frac{1}{2} U^{\mu} \bar{\sigma}_{\mu}^{\dot{\beta} \beta} D_{\alpha} D_{\beta} \bar{D}_{\dot{\beta}} X
$$

is determined so that $\delta_{\mathrm{sc}}\left[D_{\alpha} X\right]_{\mathbb{E}}$ does not contain $\bar{L}_{\dot{\alpha}}$. In fact, this transforms correctly as a (superconformal) chiral multiplet with $w=\frac{3}{2}$, as shown in Appendix. A. Notice that this modified superfield preserves the gauge invariance under (2.15). We have checked that each component of $\mathcal{W}_{\alpha}$ reproduces the correct forms of the field strength and the covariant derivative of the gaugino in Ref. [33].

Next we consider the non-Abelian case. In this case, there is no counterpart to $X$ in the Abelian case, and the gauge transformation is given by

$$
e^{V} \rightarrow \mathcal{U}\left(e^{\Lambda}\right) e^{V} \mathcal{U}\left(e^{\bar{\Lambda}}\right)
$$

Thus a naive definition of the field strength superfield,

$$
\mathcal{W}_{\alpha}^{\text {naive }} \equiv \frac{1}{4} \bar{D}^{2}\left(e^{V} D_{\alpha} e^{-V}\right),
$$

does not transform covariantly not only under the superconformal transformation, but also under the gauge transformation. We modify $\mathcal{W}_{\alpha}^{\text {naive }}$ in the same strategy, and obtain

$$
\mathcal{W}_{\alpha}=\frac{1}{4} \bar{D}^{2}\left[e^{V} D_{\alpha} e^{-V}\right]_{\mathbb{E}},
$$


where

$$
\begin{aligned}
{\left[e^{V} D_{\alpha} e^{-V}\right]_{\mathbb{E}} \equiv } & e^{V} D_{\alpha} e^{-V}-\frac{1}{2} \bar{\sigma}_{\mu}^{\dot{\beta} \beta} D_{\alpha} U^{\mu} \bar{D}_{\dot{\beta}}\left(e^{V} D_{\beta} e^{-V}\right) \\
& +i D_{\alpha} U^{\mu} e^{V} \partial_{\mu} e^{-V}-i U^{\mu} \partial_{\mu}\left(e^{V} D_{\alpha} e^{-V}\right)
\end{aligned}
$$

is determined so that its $\delta_{\mathrm{sc}}$-variation does not contain $\bar{L}_{\dot{\alpha}}$. This transforms correctly as a (superconformal) chiral multiplet with the Weyl weight 3/2, as shown in Appendix A, In fact, $\left[e^{V} D_{\alpha} e^{-V}\right]_{\mathbb{E}}$ transforms under the gauge transformation (2.19) as

$$
\left[e^{V} D_{\alpha} e^{-V}\right]_{\mathbb{E}} \rightarrow e^{\Lambda}\left[e^{V} D_{\alpha} e^{-V}\right]_{\mathbb{E}} e^{-\Lambda}+e^{\Lambda} D_{\alpha} e^{-\Lambda}
$$

so that $\mathcal{W}_{\alpha}$ transforms covariantly.

$$
\mathcal{W}_{\alpha} \rightarrow e^{\Lambda} \mathcal{W}_{\alpha} e^{-\Lambda}
$$

Therefore, (2.21) is the desired field strength superfield.

\subsection{Invariant action}

\subsection{1 $F$ - and $D$-term formulae}

Now we construct invariant actions under the gauge and superconformal transformations. First, let us consider the chiral superspace integral of a chiral superfield $W$,

$$
S_{F}[W] \equiv \int d^{2} \theta W+\text { h.c. }
$$

We can easily check that this is invariant under (2.12) when the Weyl weight of $W$ is 3 . This is the superfield description of the F-term action formula in Ref. [34].

Unlike the chiral superspace integral, the full superspace integral of a real scalar superfield $\Omega$ is not invariant by itself for any choice of the Weyl weight. An invariant action can be constructed with the aid of the gravitational superfield $U^{\mu}$ as 9

$$
S_{D}[\Omega]=2 \int d^{4} x \int d^{4} \theta\left(1+\frac{1}{3} E_{1}\right) \Omega,
$$

where the Weyl weight of $\Omega$ is 2 , and

$$
E_{1} \equiv \frac{1}{4} \bar{\sigma}_{\mu}^{\dot{\alpha} \alpha}\left[D_{\alpha}, \bar{D}_{\dot{\alpha}}\right] U^{\mu}
$$

\footnotetext{
${ }^{9}$ The factor 2 is necessary to match the normalization of the $D$-term action formula in Ref. 34.
} 
which transforms as

$$
\delta_{\mathrm{sc}} E_{1}=-\frac{1}{2} \bar{D}^{2} D^{\alpha} L_{\alpha}+\frac{3 i}{2} \sigma_{\alpha \dot{\alpha}}^{\mu} \partial_{\mu} \bar{D}^{\dot{\alpha}} L^{\alpha}+\text { h.c. . }
$$

The action (2.26) reproduces the $D$-term action formula in Ref. [34] up to linear order in the gravitational fields.

In summary, 4D SUGRA action is described by using (2.25) and (2.26) as

$$
\begin{aligned}
S^{(4 \mathrm{D})} & =S_{D}[\Omega]+S_{F}\left[\Phi_{C}^{3} W-\frac{1}{4} \mathcal{W}^{\alpha} \mathcal{W}_{\alpha}\right], \\
\Omega & =-\frac{3}{2}\left|\mathcal{U}\left(\Phi_{C}\right)\right|^{2} e^{-K / 3}
\end{aligned}
$$

where $\Phi_{C}$ is a chiral compensator superfield with $w=1$, and $K$ and $W$ are the Kähler potential and the superpotential, which consist of only the physical superfields with $w=0$.

Here we comment on the superconformal gauge-fixing. In order to obtain the usual Poincaré SUGRA from the superconformally symmetric action, we have to impose the gauge-fixing conditions to eliminate extra symmetries $\boldsymbol{D}, \boldsymbol{S}, U(1)_{A}, \boldsymbol{K}$. For example, they are given by

$$
C^{\Omega}=-\frac{3}{2}, \quad \zeta^{\Omega}=0, \quad \arg \left(\phi_{C}\right)=0, \quad b_{\mu}=0,
$$

in the unit of the Planck mass, $M_{\mathrm{Pl}}=1$. Here $\left[C^{\Omega}, \zeta^{\Omega}, \cdots\right]$ is a real general multiplet corresponding to the real superfield $\Omega$, and $\phi_{C}$ is the scalar component of the chiral multiplet corresponding to $\Phi_{C}$. The last condition is already imposed in our superfield description as mentioned in footnote 4 . The gauge-fixing (2.30) leads to the canonically normalized Einstein-Hilbert term. For a choice of $\Omega$ in (2.29), the first and third conditions in (2.30) are summarized as $\phi_{C}=\exp \left(\left.K\right|_{0} / 6\right)$.

\subsubsection{Kinetic terms for $U^{\mu}$}

Since we have neglected terms beyond linear order in $U^{\mu}$, eqs.(2.25) and (2.26) do not contain its kinetic terms. In order to deal with them, we introduce quadratic terms in $U^{\mu}$ that are independent of the matter superfields. Namely, we extend (2.26) as

$$
S_{D}[\Omega]=\int d^{4} x \int d^{4} \theta\left\{E_{2}+2\left(1+\frac{1}{3} E_{1}\right) \Omega\right\}
$$

where $E_{2}$ is quadratic in $U^{\mu}$ and independent of the matter superfields. Since $\delta_{\mathrm{sc}} E_{2}$ is linear in $U^{\mu}, E_{2}$ is determined by the requirement that a matter-independent part of $\delta_{\mathrm{sc}} S_{D}[\Omega]$ 
vanishes up to linear order in the gravitational fields. We consider a case that $\Omega$ is given by (2.29). In order to pick up the matter-independent part of $\Omega$, we expand the compensator superfield $\Phi_{C}$ around the background value $\left\langle\phi_{C}\right\rangle=\langle\exp (K / 6)\rangle$ as

$$
\Phi_{C}=\left\langle e^{K / 6}\right\rangle+\tilde{\Phi}_{C}
$$

Then, from (2.12), we have

$$
\begin{aligned}
\delta_{\mathrm{sc}} \Omega & =-\frac{3}{2}\left\langle e^{K / 6}\right\rangle\left(\delta_{\mathrm{sc}} \tilde{\Phi}_{C}+i U^{\mu} \partial_{\mu} \delta_{\mathrm{sc}} \tilde{\Phi}_{C}\right)\left\langle e^{-K / 3}\right\rangle+\text { h.c. }+\cdots \\
& =\frac{1}{8}\left(\bar{D}^{2} D^{\alpha} L_{\alpha}+\text { h.c. }\right)+\frac{1}{8} U^{\mu} \partial_{\mu}\left(i \bar{D}^{2} D^{\alpha} L_{\alpha}+\text { h.c. }\right)+\cdots
\end{aligned}
$$

Thus we can show that

$$
\begin{aligned}
\delta_{\mathrm{sc}} S_{D}[\Omega]= & \int d^{4} x \int d^{4} \theta\left\{\delta_{\mathrm{sc}} E_{2}+\frac{2}{3} \delta_{\mathrm{sc}} E_{1} \Omega+2\left(1+\frac{1}{3} E_{1}\right) \delta_{\mathrm{sc}} \Omega\right\} . \\
= & \int d^{4} x \int d^{4} \theta\left\{\delta_{\mathrm{sc}} E_{2}+\frac{1}{12} E_{1}\left(\bar{D}^{2} D^{\alpha} L_{\alpha}+\text { h.c. }\right)\right. \\
& \left.+\frac{1}{4} U^{\mu} \partial_{\mu}\left(i \bar{D}^{2} D^{\alpha} L_{\alpha}+\text { h.c. }\right)\right\}+\cdots \\
= & \int d^{4} x \int d^{4} \theta\left\{\delta_{\mathrm{sc}} E_{2}-\frac{1}{6} E_{1}\left(\bar{D}^{2} D^{\alpha} L_{\alpha}+\text { h.c. }\right)\right\}+\cdots,
\end{aligned}
$$

where the ellipses in (2.33) and (2.34) denote matter-dependent terms. We have performed partial integrals at the second equality. Therefore, $E_{2}$ must satisfy

$$
\delta_{\mathrm{sc}} E_{2}=\frac{1}{6} E_{1}\left(\bar{D}^{2} D^{\alpha} L_{\alpha}+\text { h.c. }\right) \text {. }
$$

From this condition, $E_{2}$ is identified as

$$
E_{2}=-\frac{1}{8} U_{\mu} D^{\alpha} \bar{D}^{2} D_{\alpha} U^{\mu}+\frac{1}{3} E_{1}^{2}-\left(\partial_{\mu} U^{\mu}\right)^{2} .
$$

Let us compare (2.31) with the action in Refs. [25, 37]. The action (2.31) is rewritten as

$$
\begin{aligned}
S_{D}[\Omega]=\int d^{4} x \int d^{4} \theta\{ & E_{2}-\frac{\left\langle e^{-K / 6}\right\rangle}{4} \bar{\sigma}_{\mu}^{\dot{\alpha} \alpha}\left[D_{\alpha}, \bar{D}_{\dot{\alpha}}\right] U^{\mu}\left(\tilde{\Phi}_{C}+\overline{\tilde{\Phi}}_{C}\right)-3\left|\tilde{\Phi}_{C}\right|^{2} \\
& \left.-3 i\left\langle e^{-K / 6}\right\rangle U^{\mu} \partial_{\mu}\left(\tilde{\Phi}_{C}-\overline{\tilde{\Phi}}_{C}\right)\right\}+\cdots \\
= & \int d^{4} x \int d^{4} \theta\left\{E_{2}-3\left|\tilde{\Phi}_{C}\right|^{2}+2 i\left\langle e^{-K / 6}\right\rangle \partial_{\mu} U^{\mu}\left(\tilde{\Phi}_{C}-\overline{\tilde{\Phi}}_{C}\right)\right\}+\cdots
\end{aligned}
$$

We have performed partial integrals. In Refs. [25, 37], the compensator superfield $\Sigma^{\mathrm{cp}}$ is fixed by the gauge-fixing as $\Sigma^{\mathrm{cp}}=\tilde{e}_{\mu}{ }^{\mu}+\theta^{2} F^{\Sigma}$ in our notation. From (2.3) and (2.4), on the other hand, we see that $\tilde{\Phi}_{C}=\frac{1}{3}\left\langle e^{K / 6}\right\rangle \tilde{e}_{\mu}{ }^{\mu}+\theta^{2} F^{\Phi_{C}}+\cdots$ after the gauge-fixing. The ellipsis denotes terms involving phyiscal matter fields. Thus $\Sigma^{\mathrm{cp}}$ is identified as $\Sigma^{\mathrm{cp}}=3\left\langle e^{-K / 6}\right\rangle \tilde{\Phi}_{C}$. With this and (2.36), we find that (2.37) agrees with (3.19) of Ref. [25]. 


\section{5D supergravity}

Now we consider 5D SUGRA. We assume that the background geometry is flat,

$$
d s^{2}=\eta_{\mu \nu} d x^{\mu} d x^{\nu}-d y^{2}
$$

We will extend it to the warped geometry in Sec. 4.7. The following superfield description is based on the superconformal formulation of Ref. [14]-[17].

\subsection{Decomposition into $\mathcal{N}=1$ multiplets}

The 5D superconformal transformations are devided into two parts $\delta_{\mathrm{sc}}^{(1)}$ and $\delta_{\mathrm{sc}}^{(2)}$, where $\delta_{\mathrm{sc}}^{(1)}$ forms an $\mathcal{N}=1$ subalgebra, and $\delta_{\mathrm{sc}}^{(2)}$ is the rest part. We mainly focus on $\delta_{\mathrm{sc}}^{(1)}$ in the following discussion. We will consider $\delta_{\mathrm{sc}}^{(2)}$ in Sec. 4.4. As shown in Ref. [17], each 5D superconformal multiplet can be decomposed into $\mathcal{N}=1$ superconformal multiplets, which only respect $\delta_{\mathrm{sc}}^{(1)}$ manifestly.

A hypermultiplet $\mathbb{H}^{a}\left(a=1,2, \cdots, n_{C}+n_{H}\right)$ is decomposed into two chiral multiplets $\left(\Phi^{2 a-1}, \Phi^{2 a}\right)$, and a vector multiplet $\mathbb{V}^{I}\left(I=0,1, \cdots, n_{V}\right)$ is into $\mathcal{N}=1$ vector and chiral multiplets $\left(V^{I}, \Sigma^{I}\right)$. Here $n_{C}\left(n_{H}\right)$ and $n_{V}$ are the numbers of the compensator (physical) hypermultiplets and the physical vector multiplets. These $\mathcal{N}=1$ multiplets are expressed by $\mathcal{N}=1$ superfields as explained in the previous section. The explicit forms of these superfields are listed in Appendix B. Here there is one point to notice. In the decompositions in Ref. [17], the $Z_{2}$-odd fields are dropped because such decompositions are considered only on the $S^{1} / Z_{2}$ boundaries where the $Z_{2}$-odd fields vanish, in order to describe couplings between the bulk multiplets and 4D multiplets localized on the boundaries. In this paper, on the other hand, the $\mathcal{N}=1$ decompositions are considered in the bulk. Therefore, each component of the $\mathcal{N}=1$ superfields listed in Appendix $B$ may be corrected by terms involving the $Z_{2}$-odd fields. We will come back to this point in Sec. 4.5,

The 5D Weyl multiplet $\mathbb{E}_{W}$ (or the $5 \mathrm{D}$ gravitational multiplet) is also decomposed into $\mathcal{N}=1$ multiplets. Here $\mathbb{E}_{W}$ consists of the fünfbein $e_{M} \frac{N}{M}$, the gravitini $\psi_{M}^{i}$, the $S U(2)_{U}$

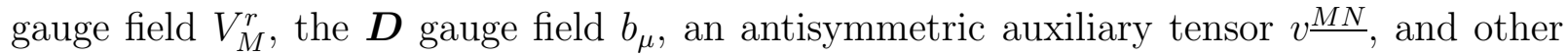
auxiliary fields. The $5 \mathrm{D}$ indices $M, N=0,1,2,3, y$ and $\underline{M}, \underline{N}=0,1,2,3,4$ denote the curved and the flat ones, and $i=1,2$ and $r=1,2,3$ denote the $S U(2)_{U}$-doublet and triplet indices. In the case that the extra dimension is compactified on $S^{1} / Z_{2}$, the $Z_{2}$-even 
part of $\mathbb{E}_{W}$ forms the $\mathcal{N}=1$ Weyl multiplet and a real general multiplet, which can be expressed by the following superfields 10

$$
\begin{gathered}
U^{\mu}=\left(\theta \sigma^{\nu} \bar{\theta}\right) \tilde{e}_{\nu}{ }^{\mu}+i \bar{\theta}^{2}\left(\theta \sigma^{\nu} \bar{\sigma}^{\mu} \psi_{\nu}^{+}\right)-i \theta^{2}\left(\bar{\theta} \bar{\sigma}^{\nu} \sigma^{\mu} \bar{\psi}_{\nu}^{+}\right)+\theta^{2} \bar{\theta}^{2}\left(V^{3 \mu}+v_{4}^{\mu}-\frac{1}{4} \epsilon^{\mu \nu \rho \tau} \partial_{\nu} \tilde{e}_{\rho \tau}\right) \\
V_{E}=\left\{1-\frac{1}{6}(\mathcal{E}+\overline{\mathcal{E}})\right\}\left\{\left(1+\tilde{e}_{y}{ }^{4}\right)+2 \theta \psi_{y}^{-}+2 \bar{\theta} \bar{\psi}_{y}^{-}-i \theta^{2}\left(V_{y}^{1}+i V_{y}^{2}\right)+i \bar{\theta}^{2}\left(V_{y}^{1}-i V_{y}^{2}\right)\right. \\
\left.-\frac{2}{3}\left(\theta \sigma^{\mu} \bar{\theta}\right)\left\{V_{\mu}^{3}-2 v_{\mu 4}\right\}+\cdots\right\}
\end{gathered}
$$

where $\tilde{e}_{M}^{N} \equiv e_{M} \frac{N}{M}-\delta_{M}^{N}$, the 2-component spinors $\psi_{M}^{ \pm}$are defined from the 4-component notation of Ref. [17] through (B.6), and

$$
\mathcal{E} \equiv \tilde{e}_{\mu}^{\mu}-2 i \theta \sigma^{\mu} \bar{\psi}_{\mu}^{+} .
$$

As mentioned in Sec. 2.1, we need not discriminate the curved and the flat indices since we keep the gravitational fields up to linear order and the background geometry is flat. Thus we again omit the underlines of the flat indices.

The $\delta_{\mathrm{sc}}^{(1)}$-transformation laws of the above superfields are the same as the $\delta_{\mathrm{sc}^{-}}$ transformation in the previous section. Namely,

$$
\begin{aligned}
\delta_{\mathrm{sc}}^{(1)} U^{\mu} & =\frac{1}{2} \sigma_{\alpha \dot{\alpha}}^{\mu}\left(\bar{D}^{\dot{\alpha}} L^{\alpha}-D^{\alpha} \bar{L}^{\dot{\alpha}}\right) \\
\delta_{\mathrm{sc}}^{(1)} V_{E} & =\left(-\frac{1}{4} \bar{D}^{2} L^{\alpha} D_{\alpha}-\frac{i}{2} \sigma_{\alpha \dot{\alpha}}^{\mu} \bar{D}^{\dot{\alpha}} L^{\alpha} \partial_{\mu}+\frac{1}{24} \bar{D}^{2} D^{\alpha} L_{\alpha}+\text { h.c. }\right) V_{E}, \\
\delta_{\mathrm{sc}}^{(1)} \Phi^{\bar{a}} & =\left(-\frac{1}{4} \bar{D}^{2} L^{\alpha} D_{\alpha}-i \sigma_{\alpha \dot{\alpha}}^{\mu} \bar{D}^{\dot{\alpha}} L^{\alpha} \partial_{\mu}-\frac{1}{8} \bar{D}^{2} D^{\alpha} L_{\alpha}\right) \Phi^{\bar{a}}, \\
\delta_{\mathrm{sc}}^{(1)} V^{I} & =\left(-\frac{1}{4} \bar{D}^{2} L^{\alpha} D_{\alpha}-\frac{i}{2} \sigma_{\alpha \dot{\alpha}}^{\mu} \bar{D}^{\dot{\alpha}} L^{\alpha} \partial_{\mu}+\text { h.c. }\right) V^{I}, \\
\delta_{\mathrm{sc}}^{(1)} \Sigma^{I} & =\left(-\frac{1}{4} \bar{D}^{2} L^{\alpha} D_{\alpha}-i \sigma_{\alpha \dot{\alpha}}^{\mu} \bar{D}^{\dot{\alpha}} L^{\alpha} \partial_{\mu}\right) \Sigma^{I},
\end{aligned}
$$

where the index $\bar{a}$ runs over the whole $2\left(n_{C}+n_{H}\right)$ chiral multiplets coming from the hypermultiplets. Notice that the Weyl weights of these superfields are $w\left(V_{E}\right)=-1, w\left(\Phi^{\bar{a}}\right)=3 / 2$, and $w\left(V^{I}\right)=w\left(\Sigma^{I}\right)=0$. Since $V_{E}$ has a nonzero background value $\left\langle V_{E}\right\rangle=1$, the gravitational fluctuation superfield is $\tilde{V}_{E} \equiv V_{E}-1$, and its transformation law should be written as

$$
\delta_{\mathrm{sc}}^{(1)} \tilde{V}_{E}=\frac{1}{24}\left(\bar{D}^{2} D^{\alpha} L_{\alpha}+\text { h.c. }\right),
$$

up to the order concerned in this paper. However, since $V_{E}$ behaves as a real general multiplet under $\delta_{\mathrm{sc}}^{(1)}$, it is sometimes convenient to treat it as a matter multiplet.

\footnotetext{
${ }^{10}$ Just like in the 4 D SUGRA case, the $\boldsymbol{D}$ gauge field $b_{\mu}$ can be set to zero, which corresponds to the $\boldsymbol{K}$ gauge fixing.
} 


\subsection{Superfield action without gravitational fluctuation modes}

In order to identify the gravitational coupling to the matter superfields, we start with the $5 \mathrm{D}$ superfield action in which the gravitational fields are fixed to their background values. Such an action was derived in Ref. [26, 27] as 11

$$
\begin{aligned}
S_{0}= & \int d^{5} x\left(\mathcal{L}_{0}^{\text {hyper }}+\mathcal{L}_{0}^{\text {vector }}\right) \\
\mathcal{L}_{0}^{\text {hyper }}= & -2 \int d^{4} \theta d_{\bar{a}}^{\bar{b}} \bar{\Phi}_{\bar{b}}\left(e^{-2 i g V^{I} t_{I}}\right)_{\bar{c}}^{\bar{a}} \Phi^{\bar{c}} \\
& +\int d^{2} \theta \Phi^{\bar{a}} d_{\bar{a}}^{\bar{b}} \rho_{\bar{b} \bar{c}}\left(\partial_{y}-2 i g \Sigma^{I} t_{I}\right)^{\bar{c}}{ }_{\bar{d}} \Phi^{\bar{d}}+\text { h.c. }, \\
\mathcal{L}_{0}^{\text {vector }}= & -\int d^{4} \theta C_{I J K} \mathcal{V}_{0}^{I} \mathcal{V}_{0}^{J} \mathcal{V}_{0}^{K} \\
& +\int d^{2} \theta \frac{3 C_{I J K}}{2}\left\{-\Sigma^{I} \mathcal{W}_{0}^{J} \mathcal{W}_{0}^{K}+\frac{1}{12} \bar{D}^{2}\left(\mathcal{Z}_{0}^{I J \alpha}\right) \mathcal{W}_{0 \alpha}^{K}\right\}+\text { h.c. },
\end{aligned}
$$

where $\bar{\Phi}_{\bar{b}} \equiv\left(\Phi^{\bar{b}}\right)^{\dagger}, d_{\bar{a}}^{\bar{b}} \equiv \operatorname{diag}\left(\mathbf{1}_{2 n_{C}},-\mathbf{1}_{2 n_{H}}\right), \rho_{\bar{a} \bar{b}} \equiv i \sigma_{2} \otimes \mathbf{1}_{n_{C}+n_{H}}$, and real constants $C_{I J K}$ are a completely symmetric tensor. The generators $t_{I}\left(I=0,1, \cdots, n_{V}\right)$ are anti-hermitian and normalized as $\operatorname{tr}\left(t_{I} t_{J}\right)=-\frac{1}{2}$. The gauge couplings $g$ can take different values for each simple or Abelian factor of the gauge group. The assumption of the 5D flat geometry (3.1) is equivalent to that the compensator hypermultiplets $\mathbb{H}^{a}\left(a=1, \cdots, n_{C}\right)$ are gauge-singlets. For simplicity, we consider a case of Abelian gauge group in the following. An extension to the non-Abelian case will be discussed in Sec. 4.3. Then the field strength superfields $\mathcal{W}_{0 \alpha}^{I}$ and $\mathcal{V}_{0}^{I}$ are defined as

$$
\begin{aligned}
\mathcal{W}_{0 \alpha}^{I} & \equiv-\frac{1}{4} \bar{D}^{2} D_{\alpha} V^{I} \\
\mathcal{V}_{0}^{I} & \equiv-\partial_{y} V^{I}+\Sigma^{I}+\bar{\Sigma}^{I} .
\end{aligned}
$$

Here and henceforth, the suffix 0 denotes quantities that the gravitational fluctuation modes are dropped. The second line in $\mathcal{L}_{0}^{\text {vector }}$ corresponds to the Chern-Simons terms, and $\mathcal{Z}_{0}^{I J \alpha}$ is defined as [21]

$$
\mathcal{Z}_{0}^{I J \alpha} \equiv V^{I} D^{\alpha} \partial_{y} V^{J}-\partial_{y} V^{I} D^{\alpha} V^{J}
$$

The gauge kinetic terms arise from the first term of the second line in $\mathcal{L}_{0}^{\text {vector }}$ after the superconformal gauge-fixing.

\footnotetext{
${ }^{11}$ The sign of the $d^{2} \theta$-integral in $\mathcal{L}_{\text {hyper }}$ is opposite to that of Ref. [26]. This stems from the sign difference in the definitions of the $F$-terms in the chiral superfields there.
} 


\section{Gravitational couplings}

Now we turn on the gravitational fluctuation fields, and specify their couplings to the matter superfields. The easiest one to obtain is couplings with $\tilde{V}_{E}=V_{E}-1$. In our previous work [26], we have already found the $V_{E}$-dependence of the action. It only appears in the $d^{4} \theta$-integral as

$$
S=-\int d^{5} x \int d^{4} \theta V_{E}\left\{2 d_{\bar{a}}^{\bar{b}} \bar{\Phi}_{\bar{b}}\left(e^{-2 i g V^{I} t_{I}}\right)_{\bar{c}}^{\bar{a}} \Phi^{\bar{c}}+C_{I J K} V_{E}^{-3} \mathcal{V}_{0}^{I} \mathcal{V}_{0}^{J} \mathcal{V}_{0}^{K}\right\}+\cdots
$$

Next we specify couplings with the other gravitational superfields. We devide the 5D action into the sectors of the hypermultiplets, the vector multiplets, and the gravitational kinetic terms.

\subsection{Hypermultiplet sector}

Let us first consider the hypermultiplet sector. We can easily specify the couplings with $U^{\mu}$ defined in (3.2) by the procedure explained in Sec. 2, Namely, replace (anti-)chiral superfields in the $d^{4} \theta$-integral with the embedded ones defined in (2.5), and modify the integrand as (2.31). Here $E_{1}$ and $E_{2}$ are constructed from $U^{\mu}$ in (3.2).

However these procedures are not enough to construct an invariant action under the $\delta_{\mathrm{sc}}^{(1)}$-transformation. Note that $\partial_{y} \Phi^{\bar{a}}$ does not transform as a chiral multiplet under $\delta_{\mathrm{sc}}^{(1)}$,

$$
\begin{aligned}
\delta_{\mathrm{sc}}^{(1)} \partial_{y} \Phi^{\bar{a}}= & \partial_{y}\left(\delta_{\mathrm{sc}}^{(1)} \Phi^{\bar{a}}\right) \\
= & \left(-\frac{1}{4} \bar{D}^{2} L^{\alpha} D_{\alpha}-i \sigma_{\alpha \dot{\alpha}}^{\mu} \bar{D}^{\dot{\alpha}} L^{\alpha} \partial_{\mu}-\frac{1}{8} \bar{D}^{2} D^{\alpha} D^{\alpha} L_{\alpha}\right) \partial_{y} \Phi^{\bar{a}} \\
& +\left(-\frac{1}{4} \bar{D}^{2} \partial_{y} L^{\alpha} D_{\alpha}-i \sigma_{\alpha \dot{\alpha}}^{\mu} \bar{D}^{\dot{\alpha}} \partial_{y} L^{\alpha} \partial_{\mu}-\frac{1}{8} \bar{D}^{2} D^{\alpha} \partial_{y} L_{\alpha}\right) \Phi^{\bar{a}} .
\end{aligned}
$$

In order to eliminate the terms involving $\partial_{y} L_{\alpha}$ in the second line, we introduce a spinor superfield $\Psi_{\alpha}$ that transforms as

$$
\delta_{\mathrm{sc}}^{(1)} \Psi_{\alpha}=-\partial_{y} L_{\alpha}
$$

and covariantize the derivative $\partial_{y}$ as

$$
\hat{\partial}_{y} \equiv \partial_{y}-\frac{1}{4} \bar{D}^{2} \Psi^{\alpha} D_{\alpha}-i \sigma_{\alpha \dot{\alpha}}^{\mu} \bar{D}^{\dot{\alpha}} \Psi^{\alpha} \partial_{\mu}-\frac{w}{12} \bar{D}^{2} D^{\alpha} \Psi_{\alpha}
$$


where $w$ is the Weyl weight of a superfield which $\hat{\partial}_{y}$ acts on. Then, $\hat{\partial}_{y} \Phi^{\bar{a}}$ transforms just in the same way as $\Phi^{\bar{a}}$ does. As a result, $\mathcal{L}_{0}^{\text {hyper }}$ is promoted as

$$
\begin{aligned}
\mathcal{L}^{\text {hyper }}= & 2 \int d^{4} \theta\left\{\frac{\Omega_{\mathrm{c}}^{\text {hyper }}}{3} E_{2}+\left(1+\frac{1}{3} E_{1}\right) \Omega^{\text {hyper }}\right\} \\
& +\int d^{2} \theta \Phi^{\bar{a}} d_{\bar{a}}^{\bar{b}} \rho_{\bar{b} \bar{c}}\left(\hat{\partial}_{y}-2 i g \Sigma^{I} t_{I}\right)_{\bar{d}}^{\bar{c}} \Phi^{\bar{d}}+\text { h.c. },
\end{aligned}
$$

where

$$
\Omega^{\text {hyper }} \equiv-V_{E} d_{\bar{a}}^{\bar{b}} \mathcal{U}\left(\bar{\Phi}_{\bar{b}}\right)\left(e^{-2 i g V^{I} t_{I}}\right)_{\bar{c}}^{\bar{a}} \mathcal{U}\left(\Phi^{\bar{c}}\right),
$$

and $\Omega_{\mathrm{c}}^{\text {hyper }}$ is a constant part of $\Omega^{\text {hyper }}$, which is determined by the $\boldsymbol{D}$ gauge fixing condition.

\subsection{Vector multiplet sector}

Next we consider the vector multiplet sector. The gauge transformation $\delta_{\text {gauge }}$ is given by

$$
\begin{aligned}
\delta_{\text {gauge }} V^{I} & =\mathcal{U}\left(\Lambda^{I}\right)+\mathcal{U}\left(\bar{\Lambda}^{I}\right), \\
\delta_{\text {gauge }} \Sigma^{I} & =\hat{\partial}_{y} \Lambda^{I}, \\
\delta_{\text {gauge }} \Phi^{\bar{a}} & =2 i g \Lambda^{I}\left(t_{I}\right)^{\bar{a}} \bar{b}_{\bar{b}} \Phi^{\bar{b}},
\end{aligned}
$$

where the transformation parameter $\Lambda^{I}$ is a chiral superfield. We can easily check that (4.5) is invariant under this transformation.

The field strength superfields $\mathcal{W}_{0 \alpha}^{I}$ should be modified as (2.17) with the aid of $U^{\mu}$.

$$
\begin{aligned}
\mathcal{W}_{\alpha}^{I} & \equiv-\frac{1}{4} \bar{D}^{2}\left\{D_{\alpha} X^{I}-\frac{1}{2} U^{\mu} \bar{\sigma}_{\mu}^{\dot{\beta} \beta} D_{\alpha} D_{\beta} \bar{D}_{\dot{\beta}} X^{I}\right\} \\
X^{I} & \equiv\left(1+\frac{1}{4} U^{\mu} \bar{\sigma}_{\mu}^{\dot{\alpha} \alpha}\left[D_{\alpha}, \bar{D}_{\dot{\alpha}}\right]\right) V^{I} .
\end{aligned}
$$

This is invariant under $\delta_{\text {gauge }}$.

Now we modify the other field strength superfield $\mathcal{V}_{0}^{I}$. First, $\Sigma^{I}$ and $\bar{\Sigma}^{I}$ in (3.7) should be replaced with $\mathcal{U}\left(\Sigma^{I}\right)$ and $\mathcal{U}\left(\bar{\Sigma}^{I}\right)$. Thus the first term in (3.7) must be modified so that its gauge transformation is $\mathcal{U}\left(\hat{\partial}_{y} \Lambda^{I}+\hat{\partial}_{y} \bar{\Lambda}^{I}\right)$. Here we redefine $\hat{\partial}_{y}$ as

$$
\hat{\partial}_{y}=\partial_{y}-\left(\frac{1}{4} \bar{D}^{2} \Psi^{\alpha} D_{\alpha}+\frac{1}{2} \bar{D}^{\dot{\alpha}} \Psi^{\alpha} \bar{D}_{\dot{\alpha}} D_{\alpha}+\frac{w+n}{24} \bar{D}^{2} D^{\alpha} \Psi_{\alpha}+\text { h.c. }\right),
$$

where $n$ is a chiral weight, i.e., the charge of $U(1)_{A} \subset S U(2)_{U}$, and $(w+n)^{\dagger}=w-n$. This reduces to the definition (4.4) when it acts on a chiral superfield. Since $X^{I}$ transforms as 
$\delta_{\text {gauge }} X^{I}=\Lambda^{I}+\bar{\Lambda}^{I}$, we find that $\delta_{\text {gauge }}\left(\hat{\partial}_{y} X^{I}\right)=\hat{\partial}_{y} \Lambda^{I}+\hat{\partial}_{y} \bar{\Lambda}^{I}$. Therefore, $\mathcal{V}_{0}^{I}$ is modified as

$$
\begin{aligned}
\mathcal{V}^{I} & \equiv\left(1-\frac{1}{4} U^{\mu} \bar{\sigma}_{\mu}^{\dot{\alpha} \alpha}\left[D_{\alpha}, \bar{D}_{\dot{\alpha}}\right]\right)\left(-\hat{\partial}_{y} X^{I}+\Sigma^{I}+\bar{\Sigma}^{I}\right) \\
& =-\left(\hat{\partial}_{y}+\frac{1}{4} \partial_{y} U^{\mu} \bar{\sigma}_{\mu}^{\dot{\alpha} \alpha}\left[D_{\alpha}, \bar{D}_{\dot{\alpha}}\right]\right) V^{I}+\mathcal{U}\left(\Sigma^{I}\right)+\mathcal{U}\left(\bar{\Sigma}^{I}\right)
\end{aligned}
$$

This transforms under $\delta_{\mathrm{sc}}^{(1)}$ in the same way as $V^{I}$ does.

Hence the $d^{4} \theta$-integral in $\mathcal{L}_{0}^{\text {vector }}$ is promoted as

$$
\mathcal{L}^{\text {vector }}=2 \int d^{4} \theta\left\{\frac{\Omega_{\mathrm{c}}^{\text {vector }}}{3} E_{2}+\left(1+\frac{1}{3} E_{1}\right) \Omega^{\text {vector }}\right\}+\left\{\int d^{2} \theta W^{\mathrm{CS}}+\text { h.c. }\right\},
$$

where

$$
\Omega^{\text {vector }}=-V_{E}^{-2} \frac{C_{I J K}}{2} \mathcal{V}^{I} \mathcal{V}^{J} \mathcal{V}^{K}
$$

and $\Omega_{\mathrm{c}}^{\text {vector }}$ is a constant part of $\Omega^{\text {vector }}$, which is determined by the $\boldsymbol{D}$ gauge fixing condition. The holomorphic function $W^{\mathrm{CS}}$ includes the Chern-Simons terms, and will be specified in the following.

Notice that there is no operation in the superconformal tensor calculus corresponding to $D_{\alpha}\left(\bar{D}_{\dot{\alpha}}\right)$. Hence promoting $\bar{D}^{2} \mathcal{Z}_{0 \alpha}^{I J}$ in (3.6) to SUGRA is a nontrivial task. We have to modify it so that it transforms just in the same way as $\mathcal{W}_{\alpha}^{I}$ under $\delta_{\mathrm{sc}}^{(1)}$, i.e., as a spinor chiral superfield with $w=\frac{3}{2}$. The strategy is similar to that we applied in Sec. 2.3. Since $\hat{\partial}_{y} X^{I}$ transforms in the same way as $X^{I}$,

$$
\left[D_{\alpha} \partial_{y} X^{I}\right]_{\mathbb{E}} \equiv D_{\alpha} \hat{\partial}_{y} X^{I}-\frac{1}{2} \bar{\sigma}_{\mu}^{\dot{\beta} \beta} U^{\mu} D_{\alpha} D_{\beta} \bar{D}_{\dot{\beta}} \partial_{y} X^{I}
$$

also transforms in the same way as $\left[D_{\alpha} X^{I}\right]_{\mathbb{E}}$ defined in (2.18). Namely, their transformation laws are given by

$$
\begin{aligned}
\delta_{\mathrm{sc}}^{(1)}\left[\mathcal{Y}_{\alpha}^{I}\right]_{\mathbb{E}}= & \left(-\frac{1}{4} \bar{D}^{2} L^{\beta} D_{\beta}-i \sigma_{\beta \dot{\beta}}^{\mu} \bar{D}^{\dot{\beta}} L^{\beta} \partial_{\mu}\right) \mathcal{Y}_{\alpha}^{I} \\
& +\left(-\frac{1}{4} D_{\alpha} \bar{D}^{2} L^{\beta}-\frac{1}{2} D_{\alpha} \bar{D}^{\dot{\beta}} L^{\beta} \bar{D}_{\dot{\beta}}\right) \mathcal{Y}_{\beta}^{I},
\end{aligned}
$$

where $\mathcal{Y}_{\alpha}^{I}=D_{\alpha} X^{I}, D_{\alpha} \partial_{y} X^{I}$. Furthermore, we define

$$
\left[\mathcal{X}^{I} \mathcal{Y}_{\alpha}^{J}\right]_{\mathbb{E}} \equiv \mathcal{X}^{I}\left[\mathcal{Y}_{\alpha}^{J}\right]_{\mathbb{E}}-\frac{1}{2} \bar{\sigma}_{\mu}^{\dot{\beta} \beta}\left(U^{\mu} D_{\beta} \bar{D}_{\dot{\beta}} \mathcal{X}^{I} \mathcal{Y}_{\alpha}^{J}+D_{\alpha} U^{\mu} \bar{D}_{\dot{\beta}} \mathcal{X}^{I} \mathcal{Y}_{\beta}^{J}\right)
$$

where $\mathcal{X}^{I}=X^{I}, \hat{\partial}_{y} X^{I}$, so that the $\bar{L}$-dependent terms in its $\delta_{\mathrm{sc}}^{(1)}$-variation are cancelled. Then we find that this also follow the same transformation law as (4.14). This indicates that $\bar{D}^{2}\left[\mathcal{X}^{I} \mathcal{Y}_{\alpha}^{J}\right]_{\mathbb{E}}$ transforms under $\delta_{\mathrm{sc}}^{(1)}$ just in the same way as $\mathcal{W}_{\alpha}^{I}$ does. 
However, (4.13) is not a unique way to promote $D_{\alpha} \partial_{y} X^{I}$. The following quantity also transforms as (4.14).

$$
\begin{aligned}
{\left[\partial_{y} D_{\alpha} X^{I}\right]_{\mathbb{E}} \equiv } & \partial_{y}\left[D_{\alpha} X^{I}\right]_{\mathbb{E}}+\left(-\frac{1}{4} \bar{D}^{2} \Psi^{\beta} D_{\beta}-i \sigma_{\beta \dot{\beta}}^{\mu} \bar{D}^{\dot{\beta}} \Psi^{\beta} \partial_{\mu}\right) D_{\alpha} X^{I} \\
& +\left(-\frac{1}{4} D_{\alpha} \bar{D}^{2} \Psi^{\beta}-\frac{1}{2} D_{\alpha} \bar{D}^{\dot{\beta}} \Psi^{\beta} \bar{D}_{\dot{\beta}}\right) D_{\beta} X^{I}
\end{aligned}
$$

The variations of the second and the third terms cancel the $\partial_{y} L$-dependent terms coming from the variation of the first term. The quantities in (4.13) and (4.16) are related to each other as

$$
\left[\partial_{y} D_{\alpha} X^{I}\right]_{\mathbb{E}}=\left[D_{\alpha} \partial_{y} X^{I}\right]_{\mathbb{E}}+\frac{1}{4}\left(\sigma_{\alpha \dot{\beta}}^{\mu} \partial_{y} U_{\mu}+\bar{D}_{\dot{\beta}} \Psi_{\alpha}-D_{\alpha} \bar{\Psi}_{\dot{\beta}}\right) D^{2} \bar{D}^{\dot{\beta}} X^{I}
$$

Therefore, the promoted form of $\mathcal{Z}_{0 \alpha}^{I J}$ is expressed as

$$
\mathcal{Z}_{\alpha}^{I J}=(1-a)\left[X^{I} D_{\alpha} \partial_{y} X^{J}\right]_{\mathbb{E}}+a\left[X^{I} \partial_{y} D_{\alpha} X^{J}\right]_{\mathbb{E}}-\left[\hat{\partial}_{y} X^{I} D_{\alpha} X^{J}\right]_{\mathbb{E}},
$$

where $a$ is a constant that cannot be determined only by the $\delta_{\mathrm{sc}}^{(1)}$-invariance of the action. As shown in Appendix C.1, we find that the gauge invariance requires that $a=1$. As a result, $W^{\mathrm{CS}}$ is expressed as

$$
\begin{aligned}
W^{\mathrm{CS}} & =\frac{3 C_{I J K}}{2}\left\{-\Sigma^{I} \mathcal{W}^{J} \mathcal{W}^{K}+\frac{1}{12} \bar{D}^{2}\left(\mathcal{Z}^{I J \alpha}\right) \mathcal{W}_{\alpha}^{K}\right\} \\
\mathcal{Z}_{\alpha}^{I J} & =\left[X^{I} \partial_{y} D_{\alpha} X^{J}\right]_{\mathbb{E}}-\left[\hat{\partial}_{y} X^{I} D_{\alpha} X^{J}\right]_{\mathbb{E}}
\end{aligned}
$$

\subsection{Non-Abelian case}

Here we consider the case of a non-Abelian gauge group. For simplicity, we assume that the whole gauge group is a simple non-Abelian group. In this case, it is convenient to use a matrix notation $(\Sigma, V) \equiv 2 i g\left(\Sigma^{I}, V^{I}\right) t_{I}$. The Lagrangian of hypermultiplet sector is written as

$$
\begin{aligned}
\mathcal{L}^{\text {hyper }} & =2 \int d^{4} \theta\left\{\frac{\Omega_{\mathrm{c}}^{\text {hyper }}}{3} E_{2}+\left(1+\frac{1}{3} E_{1}\right) \Omega^{\text {hyper }}\right\}+\left[\int d^{2} \theta W^{\text {hyper }}+\text { h.c. }\right], \\
\Omega^{\text {hyper }} & \equiv-V_{E}\left\{\mathcal{U}\left(\Phi_{\text {odd }}^{\dagger}\right) \tilde{d}\left(e^{V}\right)^{t} \mathcal{U}\left(\Phi_{\text {odd }}\right)+\mathcal{U}\left(\Phi_{\text {even }}^{\dagger}\right) \tilde{d} e^{-V} \mathcal{U}\left(\Phi_{\text {even }}\right)\right\}, \\
W^{\text {hyper }} & \equiv \Phi_{\text {odd }}^{t} \tilde{d}\left(\hat{\partial}_{y}-\Sigma\right) \Phi_{\text {even }}-\Phi_{\text {even }}^{t} \tilde{d}\left(\hat{\partial}_{y}+\Sigma^{t}\right) \Phi_{\text {odd }} .
\end{aligned}
$$

where $\tilde{d}=\operatorname{diag}\left(\mathbf{1}_{n_{C}},-\mathbf{1}_{n_{H}}\right)$, and $\Phi_{\text {odd }}$ and $\Phi_{\text {even }}$ are $\left(n_{C}+n_{H}\right)$-dimensional column vectors that consist of $\Phi^{2 a-1}$ and $\Phi^{2 a}$, respectively. This Lagrangian is invariant under the following 
gauge transformation.

$$
\begin{aligned}
& e^{V} \rightarrow e^{\mathcal{U}(\Lambda)} e^{V} e^{\mathcal{U}\left(\Lambda^{\dagger}\right)}, \quad \Sigma \rightarrow e^{\Lambda}\left(\Sigma-\hat{\partial}_{y}\right) e^{-\Lambda}, \\
& \Phi_{\text {odd }} \rightarrow\left(e^{-\Lambda}\right)^{t} \Phi_{\text {odd }}, \quad \Phi_{\text {even }} \rightarrow e^{\Lambda} \Phi_{\text {even }},
\end{aligned}
$$

if $e^{\Lambda}$ commutes with $\tilde{d}$.

Next we consider the vector multiplet sector. The index $I$ is now understood as that of the adjoint representation of the gauge group. For the constant tensor $C_{I J K}$, there is a set of hermitian matrices $\left\{T_{I}\right\}$, which satisfies [14]

$$
C_{I J K}=\frac{1}{6} \operatorname{tr}\left(T_{I}\left\{T_{J}, T_{K}\right\}\right) .
$$

In general, $\left\{T_{I}\right\}$ are not normalized, and related to the normalized anti-hermitian matrices $\left\{t_{I}\right\}$ through $t_{I}=i T_{I} / c$, where $c$ is a real constant 12 Then, an extension of $\Omega^{\text {vector }}$ in (4.11) to the non-Abelian gauge group is given by

$$
\Omega^{\text {vector }} \equiv \frac{c^{3}}{48 g^{3}} V_{E}^{-2} \operatorname{tr}\left(\mathcal{V}^{3}\right)
$$

where

$$
\mathcal{V} \equiv-\left(\hat{\partial}_{y}+\frac{1}{4} \partial_{y} U^{\mu} \bar{\sigma}_{\mu}^{\dot{\alpha} \alpha}\left[D_{\alpha}, \bar{D}_{\dot{\alpha}}\right]\right) e^{V} e^{-V}+\mathcal{U}(\Sigma)+e^{V} \mathcal{U}\left(\Sigma^{\dagger}\right) e^{-V}
$$

The $U^{\mu}-, \Psi_{\alpha}\left(\bar{\Psi}_{\dot{\alpha}}\right)$-independent part of $W^{\mathrm{CS}}$ in (4.11) is expressed as 38

$$
W_{0}^{\mathrm{CS}}=-\frac{c^{3}}{16 g^{3}} \operatorname{tr}\left[-\Sigma \mathcal{W}_{0}^{2}+\frac{1}{24} \bar{D}^{2}\left(\mathcal{Z}_{0}^{\alpha}\right)\left(\mathcal{W}_{0 \alpha}-\frac{1}{4} \mathcal{W}_{0 \alpha}^{(2)}\right)\right],
$$

Here we have taken the Wess-Zumino gauge, and thus,

$$
\begin{aligned}
\mathcal{W}_{0 \alpha} & \equiv \frac{1}{4} \bar{D}^{2}\left(e^{V} D_{\alpha} e^{-V}\right)=-\frac{1}{4} \bar{D}^{2} D_{\alpha} V+\frac{1}{8} \bar{D}^{2}\left[D_{\alpha} V, V\right], \\
\mathcal{Z}_{0}^{\alpha} & \equiv\left\{V, \partial_{y} D^{\alpha} V\right\}-\left\{\partial_{y} V, D^{\alpha} V\right\}
\end{aligned}
$$

and $\mathcal{W}_{0 \alpha}^{(2)}$ is a quadratic part of $\mathcal{W}_{0 \alpha}$, i.e., $\mathcal{W}_{0 \alpha}^{(2)} \equiv \frac{1}{8} \bar{D}^{2}\left[D_{\alpha} V, V\right]$. The couplings to the gravitational superfields $U^{\mu}$ and $\Psi_{\alpha}\left(\bar{\Psi}_{\dot{\alpha}}\right)$ can be obtained in the same manner as in the Abelian case. Namely, (4.25) is modified as

$$
W^{\mathrm{CS}}=-\frac{c^{3}}{16 g^{3}} \operatorname{tr}\left[-\Sigma \mathcal{W}^{2}+\frac{1}{24} \bar{D}^{2}\left(\mathcal{Z}^{\alpha}\right)\left(\mathcal{W}_{\alpha}-\frac{1}{4} \mathcal{W}_{\alpha}^{(2)}\right)\right],
$$

\footnotetext{
${ }^{12}$ In a case that the gauge group is a product of simple groups and Abelian groups, the constant $c$ can take different values for each simple or Abelian factor group.
} 
where $\mathcal{W}_{\alpha}$ is defined in (2.21), $\mathcal{W}_{\alpha}^{(2)}$ is its quadratic part in $V$, and

$$
\mathcal{Z}_{\alpha} \equiv\left[\left\{X, \partial_{y} D_{\alpha} X\right\}\right]_{\mathbb{E}}-\left[\left\{\partial_{y} X, D_{\alpha} X\right\}\right]_{\mathbb{E}}
$$

Here $X \equiv\left(1+\frac{1}{4} U^{\mu} \bar{\sigma}_{\mu}^{\dot{\alpha} \alpha}\left[D_{\alpha}, \bar{D}_{\dot{\alpha}}\right]\right) V$, and the definition of $[\cdots]_{\mathbb{E}}$ is similar to the Abelian case. We can see that (4.27) reduces to (4.19) when the gauge group is Abelian. However, the gauge-invariance of the action is not manifest now since we have chosen the WessZumino gauge.

\subsection{Kinetic terms for gravitational superfields}

Now we consider the kinetic terms for the gravitational superfields. Those for $U^{\mu}$ are obtained just in the same way as we did in Sec. 2.4.2. In addition to them, we can construct the following invariant Lagrangian term from (3.4) and (4.3).

$$
\begin{aligned}
& \mathcal{L}_{\mathcal{C}}=2 \int d^{4} \theta b \mathcal{C}^{\mu} \mathcal{C}_{\mu}, \\
& \mathcal{C}_{\mu} \equiv \partial_{y} U_{\mu}+\frac{1}{2} \bar{\sigma}_{\mu}^{\dot{\alpha} \alpha}\left(\bar{D}_{\dot{\alpha}} \Psi_{\alpha}-D_{\alpha} \bar{\Psi}_{\dot{\alpha}}\right)
\end{aligned}
$$

where $b$ is a real constant. Since this is invariant both under $\delta_{\mathrm{sc}}^{(1)}$ and $\delta_{\text {gauge }}$ by itself, a constant $b$ cannot be determined by those symmetries. Hence we now consider the invariance under the rest part of the 5D superconformal transformation $\delta_{\mathrm{sc}}^{(2)}$. This contains the translation along the fifth dimension $\boldsymbol{P}^{\mathbf{4}}$ and the Lorentz transformation $\boldsymbol{M}_{\boldsymbol{\mu} \boldsymbol{4}}$ that mixes $x^{\mu}$ and $y$. Comparing our gravitational superfields with the counterparts in Ref. [25], we expect that the transformation parameters of $\delta_{\mathrm{sc}}^{(2)}$ form a chiral superfield $Y=\xi^{4}+\cdots$, and a real scalar superfield $N=\left(\theta \sigma^{\mu} \bar{\theta}\right) \lambda_{\mu 4}+\cdots$, where $\xi^{4}$ and $\lambda_{\mu 4}$ are the transformation parameters for $\boldsymbol{P}^{\mathbf{4}}$ and $\boldsymbol{M}_{\boldsymbol{\mu 4}}$, respectively. This will be confirmed in the next subsection. There, we will also see that $\Psi_{\alpha}$ contains $e_{y} \underline{\underline{\mu}}$. Thus there should be another gravitational superfield that contains $e_{\mu}{ }^{4}$. From the correspondence to Ref. [25], such additional superfield is expected to be a real scalar superfield $U^{4}$ whose superconformal transformations are given by $\delta_{\mathrm{sc}}^{(1)} U^{4}=0$ and $\delta_{\mathrm{sc}}^{(2)} U^{4}=N$. 
We find the $\delta_{\mathrm{sc}}^{(2)}$-transformation laws of the $\mathcal{N}=1$ superfields as

$$
\begin{aligned}
\delta_{\mathrm{sc}}^{(2)} U^{\mu} & =0, \quad \delta_{\mathrm{sc}}^{(2)} \tilde{V}_{E}=\frac{1}{2} \partial_{y}(Y+\bar{Y}), \quad \delta_{\mathrm{sc}}^{(2)} \Psi_{\alpha}=\frac{i}{2} D_{\alpha} \tilde{N}, \quad \delta_{\mathrm{sc}}^{(2)} U^{4}=N \\
\delta_{\mathrm{sc}}^{(2)} \Phi_{\text {odd }} & =Y \partial_{y} \Phi_{\text {odd }}-\frac{i}{4} \bar{D}^{2}\left\{\tilde{N}\left(e^{-V}\right)^{t} \bar{\Phi}_{\text {even }}\right\} \\
\delta_{\mathrm{sc}}^{(2)} \Phi_{\text {even }} & =Y \partial_{y} \Phi_{\text {even }}+\frac{i}{4} \bar{D}^{2}\left\{\tilde{N} e^{V} \bar{\Phi}_{\text {odd }}\right\} \\
\delta_{\mathrm{sc}}^{(2)} e^{V} & =\frac{1}{2}(Y+\bar{Y}) \partial_{y} e^{V}+i \tilde{N}\left(\Sigma e^{V}-e^{V} \Sigma^{\dagger}\right) \\
\delta_{\mathrm{sc}}^{(2)} \Sigma & =\partial_{y}(Y \Sigma)-\frac{i}{8} \bar{D}^{2}\left(D^{\alpha} \tilde{N} D_{\alpha} e^{V} e^{-V}\right)
\end{aligned}
$$

where $(\Sigma, V)=2 i g\left(\Sigma^{I}, V^{I}\right) t_{I}$, and

$$
\tilde{N}=N-\frac{i}{2}(Y-\bar{Y})
$$

Here we modify some quantities by adding terms involving $U^{4}$. We redefine $\mathcal{U}$ in (2.5) as

$$
\mathcal{U}(\Phi)=\left(1+i U^{\mu} \partial_{\mu}+i U^{4} \partial_{y}\right) \Phi, \quad \mathcal{U}(\bar{\Phi})=\left(1-i U^{\mu} \partial_{\mu}-i U^{4} \partial_{y}\right) \bar{\Phi}
$$

for a chiral superfield $\Phi$. In the following, we assume the Abelian gauge group, for simplicity. Then we modify $X^{I}$ in (4.8) as

$$
X^{I} \equiv\left(1+\frac{1}{4} U^{\mu} \bar{\sigma}_{\mu}^{\dot{\alpha} \alpha}\left[D_{\alpha}, \bar{D}_{\dot{\alpha}}\right]\right) V^{I}-i U^{4}\left(\Sigma^{I}-\bar{\Sigma}^{I}\right)
$$

so that the gauge transformation law $\delta_{\text {gauge }} X^{I}=\Lambda^{I}+\bar{\Lambda}^{I}$ is maintained. This modification does not change the $\delta_{\mathrm{sc}}^{(1)}$-transformation law of it, and under the $\delta_{\mathrm{sc}}^{(2)}$-transformation,

$$
\delta_{\mathrm{sc}}^{(2)} X^{I}=\frac{1}{2}(Y+\bar{Y}) \partial_{y} X^{I}+\frac{1}{2}(Y-\bar{Y})\left(\Sigma^{I}-\bar{\Sigma}^{I}\right)
$$

With this definition of $X^{I}, \delta_{\mathrm{sc}}^{(2)} \mathcal{W}_{\alpha}^{I}$ does not depend on $N$. We also redefine the other field strength superfield $\mathcal{V}^{I}$ and $\mathcal{C}_{\mu}$ in (4.29) so that their $\delta_{\mathrm{sc}}^{(2)}$-transformation are independent of $N$,

$$
\begin{aligned}
\mathcal{V}^{I} \equiv & \left(1-\frac{1}{4} U^{\mu} \bar{\sigma}_{\mu}^{\dot{\alpha} \alpha}\left[D_{\alpha}, \bar{D}_{\dot{\alpha}}\right]\right)\left(-\hat{\partial}_{y} X^{I}+\Sigma^{I}+\bar{\Sigma}^{I}\right)-\left\{\frac{i}{2} D^{\alpha}\left(U^{4} \mathcal{W}_{\alpha}^{I}\right)+\text { h.c. }\right\} \\
= & -\left(\hat{\partial}_{y}+\frac{1}{4} \partial_{y} U^{\mu} \bar{\sigma}_{\mu}^{\dot{\alpha} \alpha}\left[D_{\alpha}, \bar{D}_{\dot{\alpha}}\right]\right) V^{I}+\mathcal{U}\left(\Sigma^{I}\right)+\mathcal{U}\left(\bar{\Sigma}^{I}\right)+i \partial_{y} U^{4}\left(\Sigma^{I}-\bar{\Sigma}^{I}\right) \\
& -\left(\frac{i}{2} D^{\alpha} U^{4} \mathcal{W}_{\alpha}^{I}+\text { h.c. }\right), \\
\mathcal{C}_{\mu} \equiv & \partial_{y} U_{\mu}+\frac{1}{2} \bar{\sigma}_{\mu}^{\dot{\alpha} \alpha}\left(\bar{D}_{\dot{\alpha}} \Psi_{\alpha}-D_{\alpha} \bar{\Psi}_{\dot{\alpha}}\right)+\partial_{\mu} U^{4} .
\end{aligned}
$$


The redefined field strength superfields transform as

$$
\begin{aligned}
\delta_{\mathrm{sc}}^{(2)} \mathcal{W}_{\alpha}^{I} & =\frac{1}{4} \bar{D}^{2} D_{\alpha}\left\{\frac{1}{2}(Y+\bar{Y}) \mathcal{V}^{I}\right\} \\
\delta_{\mathrm{sc}}^{(2)} \mathcal{V}^{I} & =\partial_{y}\left\{\frac{1}{2}(Y+\bar{Y}) \mathcal{V}^{I}\right\}+\left(\frac{1}{4} D^{\alpha} Y \mathcal{W}_{\alpha}^{I}+\text { h.c. }\right) \\
\delta_{\mathrm{sc}}^{(2)} \mathcal{C}_{\mu} & =\frac{i}{2} \partial_{\mu}(Y-\bar{Y}) .
\end{aligned}
$$

As shown in Appendix C.2, the $\delta_{\mathrm{sc}}^{(2)}$-variations of the $d^{4} \theta$-integral and the $d^{2} \theta$-integral parts of the Lagrangian are cancelled at the zeroth order in the gravitational superfields,

$$
\delta_{\mathrm{sc}}^{(2)}\left[2 \int d^{4} \theta \Omega+\left\{\int d^{2} \theta W+\text { h.c. }\right\}\right]=0,
$$

where $\Omega \equiv \Omega^{\text {hyper }}+\Omega^{\text {vector }}$ and $W \equiv W^{\text {hyper }}+W^{\mathrm{CS}}$. We have dropped total derivatives.

In order to determine a constant $b$ in (4.29), we now take into account linear order contributions to the matter-independent part of $\delta_{\mathrm{sc}}^{(2)} \mathcal{L}$ in the gravitational superfields, as we did in the $4 \mathrm{D}$ case discussed in Sec. 2.4.2. Here note that $U^{4}$ can be completely gauged away by the $N$-transformation. We will take such a gauge in the following. Next, in order to pick up the matter-independent part of the action, we expand chiral superfields $\Phi_{\text {odd }}$, $\Phi_{\text {even }}$ and $\Sigma^{I}$ around the backgrounds as

$$
\Phi_{\text {odd }}=\left\langle\Phi_{\text {odd }}\right\rangle+\tilde{\Phi}_{\text {odd }}, \quad \Phi_{\text {even }}=\left\langle\Phi_{\text {even }}\right\rangle+\tilde{\Phi}_{\text {even }}, \quad \Sigma^{I}=\left\langle\Sigma^{I}\right\rangle+\tilde{\Sigma}^{I} .
$$

where the backgrounds $\left\langle\Phi_{\text {odd }}\right\rangle,\left\langle\Phi_{\text {even }}\right\rangle$, and $\left\langle\Sigma^{I}\right\rangle$ are functions of only $y$, and satisfy

$$
\begin{aligned}
& \left\langle\Phi_{\text {odd }}\right\rangle^{\dagger} \tilde{d}\left\langle\Phi_{\text {odd }}\right\rangle+\left\langle\Phi_{\text {even }}\right\rangle^{\dagger} \tilde{d}\left\langle\Phi_{\text {even }}\right\rangle=1, \\
& C_{I J K}\left(2 \operatorname{Re}\left\langle\Sigma^{I}\right\rangle\right)\left(2 \operatorname{Re}\left\langle\Sigma^{J}\right\rangle\right)\left(2 \operatorname{Re}\left\langle\Sigma^{K}\right\rangle\right)=1 .
\end{aligned}
$$

These conditions come from the $\boldsymbol{D}$ gauge-fixing, which we will explain in Sec. 4.6. Then, we obtain

$$
\begin{aligned}
\delta_{\mathrm{sc}}^{(2)} \Omega^{\text {hyper }} & =-\frac{1}{2} \partial_{y}(Y+\bar{Y})+\cdots \\
\delta_{\mathrm{sc}}^{(2)} \Omega^{\text {vector }} & =-\frac{1}{4} \partial_{y}(Y+\bar{Y})+\frac{3 i}{4} \partial_{y}(Y-\bar{Y}) \partial_{\mu} U^{\mu}+\cdots \\
\delta_{\mathrm{sc}}^{(2)} W^{\text {hyper }} & =\frac{i}{8} \bar{D}^{2}\left(\partial^{\mu} \bar{Y} \bar{\sigma}_{\mu}^{\dot{\alpha} \alpha} \bar{D}_{\dot{\alpha}} \Psi_{\alpha}\right)+\cdots \\
\delta_{\mathrm{sc}}^{(2)} W^{\mathrm{CS}} & =0+\cdots
\end{aligned}
$$

where the ellipses denote matter-dependent terms and higher-order terms in the gravitational superfields. We have used (4.39). The $\Psi$-dependent term appears since $\partial_{y}$ in 
$\delta_{\text {sc }}^{(2)} W^{\text {hyper }}$ in (‥15) should be understood as $\hat{\partial}_{y}$ when we take into account the linear order contributions in the gravitational superfields. Thus the $\delta_{\mathrm{sc}}^{(2)}$-variation of the 5D Lagrangian with (4.29),

$$
\mathcal{L}=\int d^{4} \theta\left\{E_{2}+2 b \mathcal{C}^{\mu} \mathcal{C}_{\mu}+2\left(1+\frac{1}{3} E_{1}\right) \Omega\right\}+\left\{\int d^{2} \theta W+\text { h.c. }\right\},
$$

is calculated as

$$
\begin{aligned}
& \delta_{\mathrm{sc}}^{(2)} \mathcal{L}=\int d^{4} \theta[ 4 b \mathcal{C}^{\mu} \delta_{\mathrm{sc}}^{(2)} \mathcal{C}_{\mu}-\frac{1}{2} \partial_{y}(Y+\bar{Y}) E_{1} \\
&\left.+\frac{i}{2} \partial^{\mu}(Y-\bar{Y})\left\{3 \partial_{y} U_{\mu}+\bar{\sigma}_{\mu}^{\dot{\alpha} \alpha}\left(\bar{D}_{\dot{\alpha}} \Psi_{\alpha}-D_{\alpha} \bar{\Psi}_{\dot{\alpha}}\right)\right\}\right]+\cdots \\
&=\int d^{4} \theta\left[\frac{i}{2} \partial^{\mu}(Y-\bar{Y})\left(4 b \mathcal{C}_{\mu}+2 \mathcal{C}_{\mu}\right)\right]+\cdots
\end{aligned}
$$

where the ellipsis denote matter-dependent terms and higher-order terms in the gravitational superfields. We have dropped total derivatives. Therefore, the $\delta_{\mathrm{sc}}^{(2)}$-invariance of the action requires that $b=-\frac{1}{2}$.

\subsection{Identification of components in $\Psi_{\alpha}$ and $U^{4}$}

In this subsection, we identify the components in the $Z_{2}$-odd gravitational superfields $\Psi_{\alpha}$ and $U^{4}$ with the component fields in Ref. [17]. By comparing (4.30) with the superconformal transformations in Ref. [17], we identify components of $Y$ and $N$ as

$$
\begin{aligned}
Y= & \xi^{4}+4 \theta \epsilon_{\alpha}^{-}+i \theta^{2}\left(\vartheta_{V}^{1}+i \vartheta_{V}^{2}\right), \\
N= & \left(\theta \sigma^{\mu} \bar{\theta}\right)\left(\lambda_{\mu 4}+\partial_{\mu} \xi^{4}\right)+\theta^{2} \bar{\theta}\left(\bar{\eta}^{+}-\frac{1}{4} \bar{\sigma}^{\mu} \partial_{\mu} \epsilon^{-}\right)+\bar{\theta}^{2} \theta\left(\eta^{+}+\frac{1}{4} \sigma^{\mu} \partial_{\mu} \bar{\epsilon}^{-}\right) \\
& +\cdots,
\end{aligned}
$$

where we take the chiral coordinate $y^{\mu}$ for $Y$. The parameters $\xi^{4}, \lambda_{\mu 4}$, and $\vartheta_{V}^{r}(r=1,2)$ are the transformation parameters for $\boldsymbol{P}^{\mathbf{4}}, \boldsymbol{M}_{\boldsymbol{\mu} \mathbf{4}}$, and $S U(2)_{U} / U(1)_{A}$. The spinors $\epsilon_{\alpha}^{-}$and $\eta_{\alpha}^{+}$are the $Z_{2}$-odd components of the transformation parameters for $\boldsymbol{Q}$ and $\boldsymbol{S}$.

Therefore, each component of $\Psi_{\alpha}$ and $U^{4}$ are identified as

$$
\begin{aligned}
\left.\bar{D}_{\dot{\alpha}} \Psi_{\alpha}\right|_{0} & =-\frac{i}{2} \sigma_{\alpha \dot{\alpha}}^{\mu} e_{y \underline{\mu}},\left.\quad D^{\alpha} \bar{D}_{\dot{\alpha}} \Psi_{\alpha}\right|_{0}=\frac{3 i}{2}\left(\psi_{\mu}^{-} \sigma^{\mu}\right)_{\dot{\alpha}}+4 \bar{\psi}_{y \dot{\alpha}}^{+}, \\
\left.D^{2} \bar{D}_{\dot{\alpha}} \Psi_{\alpha}\right|_{0} & =-4 \sigma_{\alpha \dot{\alpha}}^{\mu}\left(V_{\mu}^{1}+i V_{\mu}^{2}\right), \quad \cdots
\end{aligned}
$$

and

$$
U^{4}=\left(\theta \sigma^{\mu} \bar{\theta}\right) e_{\mu}{ }^{4}+\frac{1}{4} \bar{\theta}^{2}\left(\theta \sigma^{\mu} \bar{\psi}_{\mu}^{-}\right)-\frac{1}{4} \theta^{2}\left(\bar{\theta} \bar{\sigma}^{\mu} \psi_{\mu}^{-}\right)+\cdots
$$


Notice that $\Psi_{\alpha}$ appears in the action only through $\bar{D}_{\dot{\alpha}} \Psi_{\alpha}$ and its derivatives. Thus $\left.\Psi_{\alpha}\right|_{0}$ and $\left.D_{\alpha} \Psi_{\beta}\right|_{0}$ are irrelevant to the physics.

There is one comment on the component identification for the matter superfields. As mentioned in Sec. 3.1, each component of the $\mathcal{N}=1$ superfields listed in Appendix B may be corrected by terms involving the $Z_{2}$-odd fields. In fact, we need such correction terms in order to reproduce the correct superconformal transformations in Ref. [17]. For example, $\zeta_{\alpha}^{a \pm}$ in (B.1) must be modified as

$$
\begin{gathered}
\zeta_{\alpha}^{a-} \rightarrow \zeta_{\alpha}^{a-}-\frac{7}{8}\left(\sigma^{\mu} \bar{\psi}_{\mu}^{-}\right)_{\alpha} \overline{\mathcal{A}}_{2}^{2 a} \\
\zeta_{\alpha}^{a+} \rightarrow \zeta_{\alpha}^{a+}+\frac{7}{8}\left(\sigma^{\mu} \bar{\psi}_{\mu}^{-}\right)_{\alpha} \overline{\mathcal{A}}_{2}^{2 a-1}
\end{gathered}
$$

\subsection{Elimination of unphysical modes}

Since our action is based on the superconformal formulation, there are unphysical degrees of freedom to eliminate.

As pointed out in Ref. [29], $V_{E}$ does not have a kinetic term and can be integrated out 13 From (4.1), $V_{E}$ is expressed as

$$
V_{E}=\left(\frac{\Omega^{\mathrm{v}}}{\Omega^{\mathrm{h}}}\right)^{1 / 3}
$$

where

$$
\begin{aligned}
& \Omega^{\mathrm{h}} \equiv-\left.\Omega^{\text {hyper }}\right|_{V_{E}=1}=\mathcal{U}\left(\Phi_{\text {odd }}^{\dagger}\right) \tilde{d}\left(e^{V}\right)^{t} \mathcal{U}\left(\Phi_{\text {odd }}\right)+\mathcal{U}\left(\Phi_{\text {even }}^{\dagger}\right) \tilde{d} e^{-V} \mathcal{U}\left(\Phi_{\text {even }}\right), \\
& \Omega^{\mathrm{v}} \equiv-\left.2 \Omega^{\text {vector }}\right|_{V_{E}=1}=C_{I J K} \mathcal{V}^{I} \mathcal{V}^{J} \mathcal{V}^{K}=-\frac{c^{3}}{24 g^{3}} \operatorname{tr}\left(\mathcal{V}^{3}\right)
\end{aligned}
$$

After integrating it out, $\Omega$ in the action becomes

$$
\Omega=-\frac{3}{2}\left(\Omega^{\mathrm{h}}\right)^{2 / 3}\left(\Omega^{\mathrm{v}}\right)^{1 / 3} .
$$

In order to obtain the Poincaré SUGRA, we have to impose the superconformal gauge fixing conditions to eliminate the extra symmetries. The following conditions imposed on the hypermultiplet sector fix those symmetries.

$$
\begin{aligned}
d_{\bar{a}}^{\bar{b}} \bar{\phi}_{\bar{b}} \phi^{\bar{a}} & =\phi_{\text {odd }}^{\dagger} \tilde{d} \phi_{\text {odd }}+\phi_{\text {even }}^{\dagger} \tilde{d} \phi_{\text {even }}=1, \\
d_{\bar{a}} \bar{\phi}_{\bar{b}} \chi^{\bar{a}} & =\phi_{\text {odd }}^{\dagger} \tilde{d} \chi_{\text {odd }}+\phi_{\text {even }}^{\dagger} \tilde{d} \chi_{\text {even }}=0, \\
\phi^{\bar{a}} d_{\bar{a}}^{\bar{b}} \rho_{\bar{b} \bar{c}} \chi^{\bar{c}} & =\phi_{\text {odd }} \tilde{d} \chi_{\text {even }}-\phi_{\text {even }} \tilde{d} \chi_{\text {odd }}=0, \\
\phi^{1} & =0, \quad \arg \left(\phi^{2}\right)=0,
\end{aligned}
$$

\footnotetext{
${ }^{13}$ This does not mean that $e_{y}{ }^{4}$ is an auxiliary field. It is also contained in $\Sigma^{I}$, which have their own kinetic terms.
} 
in the unit of the 5D Planck mass $M_{5}=1$. Here $\left[\phi^{\bar{a}}, \chi^{\bar{a}}, \cdots\right]$ is a chiral multiplet corresponding to a chiral superfield $\Phi^{\bar{a}}$. The first condition fixes the $\boldsymbol{D}$ gauge, the second and the third fix the $\boldsymbol{S}$ gauge, and the fourth and the fifth fix the $S U(2)_{U}$ gauge. The $\boldsymbol{K}$ gauge is already fixed in our formalism. In the single compensator case $n_{C}=1$, for example, They are rewritten as

$$
\begin{aligned}
& \phi^{1}=0, \quad \phi^{2}=\left\{1+\sum_{\bar{a}=3}^{2\left(n_{H}+1\right)}\left|\phi^{\bar{a}}\right|^{2}\right\}^{1 / 2}, \\
& \chi_{\alpha}^{1}=-\left(\phi^{2}\right)^{-1} \sum_{\bar{a}, \bar{b}=3}^{2\left(n_{H}+1\right)} \rho_{\bar{a} \bar{b}} \phi^{\bar{a}} \chi_{\alpha}^{\bar{b}}, \quad \chi_{\alpha}^{2}=\left(\bar{\phi}^{2}\right)^{-1} \sum_{\bar{a}=3}^{2\left(n_{H}+1\right)} \bar{\phi}^{\bar{a}} \chi^{\bar{a}} .
\end{aligned}
$$

The components of the compensator hypermultiplet are now expressed in terms of the physical fields.

Instead of the first two conditions in (4.50), we can also take the following gauge fixing conditions.

$$
\left.\Omega^{\mathrm{h}}\right|_{0}=1,\left.\quad D_{\alpha} \Omega^{\mathrm{h}}\right|_{0}=0 .
$$

Notice that these are different from (4.50) due to the existence of $\mathcal{E}$ in (2.3). These conditions can be rewritten as

$$
\left.\frac{\Omega^{\mathrm{v}}}{V_{E}^{3}}\right|_{0}=1,\left.\quad D_{\alpha}\left(\frac{\Omega^{\mathrm{v}}}{V_{E}^{3}}\right)\right|_{0}=0,
$$

or

$$
\left.\Omega\right|_{0}=-\left.\frac{3}{2} V_{E}\right|_{0},\left.\quad D_{\alpha} \Omega\right|_{0}=-\left.\frac{3}{2} D_{\alpha} V_{E}\right|_{0}
$$

We have used (4.47).

The expression (4.49) is useful to compare our superfield Lagrangian to that of Ref. [25]. For simplicity, we consider the single compensator case. In this case, $\Phi^{2}$ plays a similar role to the chiral compensator multiplet in 4D SUGRA. To emphasize this point, we redefine $\Phi^{\bar{a}}$ as $\Phi_{C} \equiv\left(\Phi^{2}\right)^{2 / 3}$ and $\hat{\Phi}^{\bar{a}} \equiv \Phi^{\bar{a}} / \Phi^{2}(\bar{a} \neq 2)$ so that their Weyl weights are one and zero, respectively. In a case that the background values of physical matter fields are zero or negligible compared with $M_{5}$, (4.38) is rewritten as

$$
\Phi_{C}=1+\tilde{\Phi}_{C}, \quad \hat{\Phi}^{\bar{a}}=0+\cdots, \quad \Sigma^{I}=\left\langle\Sigma^{I}\right\rangle+\tilde{\Sigma}^{I},
$$

where $\bar{a} \neq 2$, and the ellipsis denotes terms involving the physical matter fields. Then, (4.49) is rewritten as

$$
\begin{aligned}
\Omega & =-\frac{3}{2}\left|\mathcal{U}\left(\Phi_{C}\right)\right|^{2}(1+\cdots)^{2 / 3}\left\{1+8 C_{I J K}\left(\operatorname{Re}\left\langle\Sigma^{I}\right\rangle\right)\left(\operatorname{Re}\left\langle\Sigma^{J}\right\rangle\right) \operatorname{Re} \mathcal{U}\left(\tilde{\Sigma}^{K}\right)+\cdots\right\} \\
& =-\frac{3}{2}\left|1+\tilde{\Phi}_{C}+i U^{\mu} \partial_{\mu} \tilde{\Phi}_{C}\right|^{2}\left\{1+T+\bar{T}+i U^{\mu} \partial_{\mu}(T-\bar{T})+\cdots\right\}
\end{aligned}
$$


where $T \equiv 4 C_{I J K}\left(\operatorname{Re}\left\langle\Sigma^{I}\right\rangle\right)\left(\operatorname{Re}\left\langle\Sigma^{J}\right\rangle\right) \tilde{\Sigma}^{K}$ is (the fluctuation of) the 5D radion superfield [32]. Then the 5D Lagrangian is

$$
\begin{array}{r}
\mathcal{L}=\int d^{4} \theta\left\{E_{2}-\mathcal{C}^{\mu} \mathcal{C}_{\mu}+2\left(1+\frac{1}{3} E_{1}\right) \Omega\right\}+\cdots \\
=\int d^{4} \theta\left[E_{2}-\mathcal{C}^{\mu} \mathcal{C}_{\mu}-3\left|\tilde{\Phi}_{C}\right|^{2}-3 i U^{\mu} \partial_{\mu}\left(\tilde{\Phi}_{C}+T+\text { h.c. }\right)\right. \\
\left.\quad-E_{1}\left(\tilde{\Phi}_{C}+T+\text { h.c. }\right)-3\left(\overline{\tilde{\Phi}}_{C} T+\text { h.c. }\right)\right]+\cdots \\
=\int d^{4} \theta\left[\begin{array}{l}
E_{2}-3\left|\tilde{\Phi}_{C}\right|^{2}+2 i \partial_{\mu} U^{\mu}\left(\tilde{\Phi}_{C}-\overline{\tilde{\Phi}}_{C}\right) \\
\left.\quad-\mathcal{C}^{\mu} \mathcal{C}_{\mu}+\left\{\left(-3 \overline{\tilde{\Phi}}_{C}+2 i \partial_{\mu} U^{\mu}\right) T+\text { h.c. }\right\}\right]+\cdots
\end{array}\right.
\end{array}
$$

We have dropped total derivatives. Recalling that the chiral compensator $\Sigma^{\mathrm{cp}}$ in Ref. [25] is identified with $\Sigma^{\mathrm{cp}}=3 \tilde{\Phi}_{C}$, this agrees with (3.19) and (3.20) of Ref. [25], except for the second line in (3.20) of Ref. [25]. There is no counterpart of the latter in our formulation because we do not need the prepotential for $\Sigma^{\mathrm{cp}}$ to construct an invariant action. In Ref. [25], the role of the second line in (3.20) is to cancel the $\delta_{\mathrm{sc}}^{(2)}$-variation of the first and the third lines. In our formulation, such variation is cancelled by $\delta_{\mathrm{sc}}^{(2)} \Omega$ and $\delta_{\mathrm{sc}}^{(2)} W$ in (4.40).

\subsection{Case of warped geometry}

We have assumed the 5D flat spacetime (3.1) as a background geometry. Here we extend the results obtained so far to a warped geometry whose metric is given by

$$
d s^{2}=e^{2 \sigma(y)} \eta_{\mu \nu} d x^{\mu} d x^{\nu}-d y^{2}
$$

This is the most generic metric that has the 4D Poincaré symmetry. The warp factor $\sigma(y)$ is determined by solving the equations of motion. Especially, for a case of a supersymmetric background, it is obtained as a solution to the BPS equations. (See, for example, Ref. [30].) A nontrivial warp factor is obtained in a case that the compensator hypermultiplets are charged under some of the gauge groups, i.e., the gauged SUGRA [3, 39, 40]. The warp factor can be easily taken into account in the following way. First we rescale each component field by $e^{-w \sigma}$, where $w$ is its Weyl weight [27]. Second we change the coordinate $y$ as

$$
d \hat{y}=e^{-\sigma(y)} d y
$$

Then, the above background metric becomes the flat one,

$$
d s^{2}=\eta_{\mu \nu} d x^{\mu} d x^{\nu}-d \hat{y}^{2}
$$


This means that we can obtain the superfield action in the warped spacetime from that in the flat spacetime by going back to the $y$-coordinate and rescaling each component field by $e^{w \sigma}$. For example, $e_{y}^{4}$ transforms under such transformations as

$$
e_{y}^{4} \rightarrow e^{\sigma} e_{y}^{4} \rightarrow e_{y}^{4}
$$

where the first transformation is by $\hat{y} \rightarrow y$ and the second one is the Weyl rescaling. Thus the background value of $V_{E}$ remains to be one after these transformations. It is convenient to further rescale the chiral superfields $\Phi^{\bar{a}}$ and $\Sigma^{I}$ as

$$
\Phi^{\bar{a}} \rightarrow e^{\frac{3}{2} \sigma} \Phi^{\bar{a}}, \quad \Sigma^{I} \rightarrow e^{\sigma} \Sigma^{I},
$$

so that the lowest components of the superfields do not contain the warp factor. Then the $\boldsymbol{D}$ gauge fixing condition in (4.52) or (4.53) is unchanged. The covariant derivative $\hat{\partial}_{y}$ transforms as $\hat{\partial}_{y} \rightarrow e^{\sigma} \hat{\partial}_{y}$, where it is now defined as

$$
\hat{\partial}_{y} \equiv \partial_{y}-e^{-\sigma}\left(\frac{1}{4} \bar{D}^{2} \Psi^{\alpha} D_{\alpha}+\frac{1}{2} \bar{D}^{\dot{\alpha}} \Psi^{\alpha} \bar{D}_{\dot{\alpha}} D_{\alpha}+\frac{w+n}{24} \bar{D}^{2} D^{\alpha} \Psi_{\alpha}+\text { h.c. }\right) .
$$

Notice that

$$
\Omega \equiv-V_{E} \Omega^{\mathrm{h}}-\frac{1}{2} V_{E}^{-2} \Omega^{\mathrm{v}}
$$

transforms as $\Omega \rightarrow e^{3 \sigma} \Omega$. This indicates that the coefficient of the kinetic terms for the SUGRA superfields is also rescaled by $e^{3 \sigma}$. As a result, the 5D Lagrangian is

$$
\begin{aligned}
\mathcal{L}= & \int d^{4} \theta e^{2 \sigma}\left\{E_{2}-\mathcal{C}^{\mu} \mathcal{C}_{\mu}+2\left(1+\frac{1}{3} E_{1}\right) \Omega\right\} \\
& +\left[\int d^{2} \theta\left(e^{3 \sigma} W^{\text {hyper }}+W^{\mathrm{CS}}\right)+\text { h.c. }\right],
\end{aligned}
$$

where

$$
\mathcal{C}_{\mu} \equiv e^{\sigma} \partial_{y} U_{\mu}+\frac{1}{2} \bar{\sigma}_{\mu}^{\dot{\alpha} \alpha}\left(\bar{D}_{\dot{\alpha}} \Psi_{\alpha}-D_{\alpha} \bar{\Psi}_{\dot{\alpha}}\right),
$$

and the definitions of the other quantities are unchanged, except for $\hat{\partial}_{y}$ defined in (4.63). We have taken into account the warp factor coming from the integral measure (4.59).

\section{Summary}

We have completed an $\mathcal{N}=1$ superfield action for the generic system of vector multiplets and hypermultiplets coupled to 5D SUGRA, based on the superconformal formulation [14-[17]. Especially we specified couplings of the gravitational superfields to the 
matter superfields, up to linear order in the former. The gravitational superfields consist of four superfields $\left(U^{\mu}, \tilde{V}_{E}, \Psi_{\alpha}, U^{4}\right)$, which correspond to the fluctuation modes around the background metric (3.1) or (4.58). The dependence of the action on these superfields is uniquely determined by the invariance under the 5D superconformal transformations $\delta_{\mathrm{sc}}^{(1)}$, $\delta_{\mathrm{sc}}^{(2)}$, and the (super)gauge transformation $\delta_{\text {gauge }}$, which are expressed in the $\mathcal{N}=1$ superfield description. Among them, $\mathcal{N}=1$ part of the $5 \mathrm{D}$ superconformal transformation $\delta_{\mathrm{sc}}^{(1)}$ mainly restricts the form of the action. The others are used to fix the coefficients $a$ in (4.18) and $b$ in (4.29). Since $\Psi_{\alpha}$ and $U^{4}$ can be gauged away by the $\delta_{\mathrm{sc}}^{(1)}$ - and $\delta_{\mathrm{sc}}^{(2)}$-transformations,

respectively, and $\tilde{V}_{E}$ can be integrated out as in (4.47), only $U^{\mu}$ remains in the unitary gauge. Notice that the matter multiplets need the help of the gravitational fields to form $\mathcal{N}=1$ superfields $\Phi^{\bar{a}}, V^{I}$ and $\Sigma^{I}$, and thus they contain the gravitational fields. This means that the kinetic terms for the gravitational superfields, i.e., the first two terms in the first line of (4.65), do not reproduce the Einstein-Hilbert term by themselves.

This work can be understood as an extension of the 5D linearized SUGRA [25] to a case that the matter superfields also propagate in the bulk. The 5D linearized SUGRA is useful to calculate quantum effects, keeping the $\mathcal{N}=1$ superfield structure [41, 42]. Our result makes it possible to perform such calculations in more generic 5D SUGRA. For example, one-loop corrections to the effective 4D Kähler potential have to be calculated when we discuss the stabilization of the size of the extra dimension by the Casimir energy [43, 44] in the context of 5D SUGRA. An investigation of such moduli stabilization by means of the superfield action obtained in this paper is one of our future projects.

Another direction to proceed is an extension of our formalism to higher-dimensional SUGRA. Although such theories do not have a full superconformal description, the $\mathcal{N}=1$ superfield description is possible. (See Ref. [21, 45], in the global SUSY case.) Such superfield description provides a powerful tool to discuss SUSY brane-world models.

\section{Acknowledgements}

This work was supported in part by Grant-in-Aid for Young Scientists (B) No.22740187 from Japan Society for the Promotion of Science.

\section{A Transformation law of field strength superfield}

Here we show that the field strength superfields defined by (2.17) and (2.21) correctly transform as (superconformal) chiral multiplets with the Weyl weight 3/2. 
First, consider the Abelian case. From (2.12) and (2.17), we find that

$$
\delta_{\mathrm{sc}} \mathcal{W}_{\alpha}=\left(-\frac{1}{4} \bar{D}^{2} L^{\beta} D_{\alpha}+\frac{1}{4} \bar{D}^{2} L_{\alpha} D^{\beta}-i \sigma_{\alpha \dot{\beta}}^{\mu} \bar{D}^{\dot{\beta}} L^{\beta} \partial_{\mu}-i \bar{\sigma}^{\mu \dot{\beta} \beta} \bar{D}_{\dot{\beta}} L_{\alpha} \partial_{\mu}+\frac{1}{4} \bar{D}^{2} D_{\alpha} L^{\beta}\right) \mathcal{W}_{\beta}
$$

and $\bar{L}_{\dot{\alpha}}$-dependent terms are certainly cancelled. Here we have used that $D^{\beta} \mathcal{W}_{\beta}=\bar{D}_{\dot{\beta}} \overline{\mathcal{W}}^{\dot{\beta}}$ and $\bar{D}^{2} \overline{\mathcal{W}}^{\dot{\beta}}=-4 i \bar{\sigma}^{\mu \dot{\beta} \beta} \partial_{\mu} \mathcal{W}_{\beta}$ at the zeroth order in $U^{\mu}$.

In general, a matrix $T_{\alpha}{ }^{\beta}$ can be expanded as

$$
T_{\alpha}{ }^{\beta}=\frac{1}{2} T_{\gamma}^{\gamma} \delta_{\alpha}{ }^{\beta}-\operatorname{Re}\left\{\left(\sigma_{\mu \nu}\right)_{\gamma}{ }^{\delta} T_{\delta}^{\gamma}\right\}\left(\sigma^{\mu \nu}\right)_{\alpha}{ }^{\beta} .
$$

Thus,

$$
T_{\alpha}{ }^{\beta}-T_{\alpha}^{\beta}=T_{\alpha}{ }^{\beta}-\epsilon^{\beta \gamma} \epsilon_{\alpha \delta} T_{\gamma}{ }^{\delta}=T_{\gamma}^{\gamma} \delta_{\alpha}{ }^{\beta},
$$

since $\epsilon^{\beta \gamma} \epsilon_{\alpha \delta}\left(\sigma^{\mu \nu}\right)_{\gamma}^{\delta}=\left(\sigma^{\mu \nu}\right)_{\alpha}^{\beta}$. Therefore,

$$
\begin{aligned}
& -\frac{1}{4} \bar{D}^{2} L^{\beta} D_{\alpha}+\frac{1}{4} \bar{D}^{2} L_{\alpha} D^{\beta}=-\frac{1}{4} \bar{D}^{2} L^{\gamma} D_{\gamma} \delta_{\alpha}{ }^{\beta}, \\
& -i \sigma_{\alpha \dot{\alpha}}^{\mu} \bar{D}^{\dot{\alpha}} L^{\beta}-i \bar{\sigma}^{\mu \dot{\beta} \beta} \bar{D}_{\dot{\beta}} L_{\alpha}=-i \epsilon^{\beta \delta} \bar{D}^{\dot{\gamma}}\left(\sigma_{\alpha \dot{\gamma}}^{\mu} L_{\delta}-\sigma_{\delta \dot{\gamma}}^{\mu} L_{\alpha}\right)=-i \sigma_{\gamma \dot{\gamma}}^{\mu} \bar{D}^{\dot{\gamma}} L^{\gamma} \delta_{\alpha}{ }^{\beta}, \\
& -\frac{1}{4} \bar{D}^{2} D_{\alpha} L^{\beta}=\frac{1}{8} \bar{D}^{2} D_{\gamma} L^{\gamma} \delta_{\alpha}{ }^{\beta}-\operatorname{Re}\left\{\left(\sigma_{\mu \nu}\right)_{\gamma}{ }^{\delta} \cdot \frac{1}{4} \bar{D}^{2} D_{\delta} L^{\gamma}\right\}\left(\sigma^{\mu \nu}\right)_{\alpha}{ }^{\beta} .
\end{aligned}
$$

By using these, (A.1) is rewritten as

$$
\begin{aligned}
\delta_{\mathrm{sc}} \mathcal{W}_{\alpha}= & \left\{-\frac{1}{4} \bar{D}^{2} L^{\beta} D_{\beta}-i \sigma_{\beta \dot{\beta}}^{\mu} \bar{D}^{\dot{\beta}} L^{\beta} \partial_{\mu}-\frac{1}{8} \bar{D}^{2} D^{\beta} L_{\beta}\right\} \mathcal{W}_{\alpha} \\
& +\frac{1}{4} \operatorname{Re}\left\{\left(\sigma_{\mu \nu}\right)_{\beta}^{\gamma} \bar{D}^{2} D^{\beta} L_{\gamma}\right\}\left(\sigma^{\mu \nu} \mathcal{W}\right)_{\alpha} .
\end{aligned}
$$

This is the correct superconformal transformation law of a chiral multiplet. The last term of the first line implies that $\mathcal{W}_{\alpha}$ have the Weyl weight $3 / 2$, and the second line is a term proportional to the Lorentz generator, which is absent in the case of scalar superfields. The chirality of the last term is not manifest, but it is ensured by the last equation in (A.4). Namely, the $\bar{\theta}^{\dot{\alpha}}$-dependent terms are cancelled by summing over the indices $\mu$ and $\nu$.

We can check the non-Abelian case in a similar way. From (2.12), we obtain

$$
\begin{aligned}
\delta_{\mathrm{sc}}\left[e^{V} D_{\alpha} e^{-V}\right]_{\mathbb{E}}= & \left(-\frac{1}{4} \bar{D}^{2} L^{\beta} D_{\beta}-i \sigma_{\beta \dot{\beta}}^{\mu} \bar{D}^{\dot{\beta}} L^{\beta} \partial_{\mu}\right)\left(e^{V} D_{\alpha} e^{-V}\right)-\frac{1}{4} \bar{D}^{2} D_{\alpha} L^{\beta} e^{V} D_{\alpha} e^{-V} \\
& -\frac{1}{2} D_{\alpha} \bar{D}^{\dot{\beta}} L^{\beta} \bar{D}_{\dot{\beta}}\left(e^{V} D_{\beta} e^{-V}\right)-i \sigma_{\alpha \dot{\beta}}^{\mu} \partial_{\mu} \bar{D}^{\dot{\beta}} L^{\beta} e^{V} D_{\beta} e^{-V}
\end{aligned}
$$

which leads to

$$
\delta_{\mathrm{sc}} \mathcal{W}_{\alpha}=\left(-\frac{1}{4} \bar{D}^{2} L^{\beta} D_{\beta}-i \sigma_{\beta \dot{\beta}}^{\mu} \bar{D}^{\dot{\beta}} L^{\beta} \partial_{\mu}\right) \mathcal{W}_{\alpha}+\frac{1}{4} \bar{D}^{2} D_{\alpha} L^{\beta} \mathcal{W}_{\beta}
$$

With the last equation in (A.4), this is the same transformation law as (A.5). 


\section{B Explicit forms of $\mathcal{N}=1$ superfields in 5D SUGRA}

Here we collect explicit expressions of $\mathcal{N}=1$ superfields originating from 5D hyper and vector multiplets. We omit terms involving the $Z_{2}$-odd gravitational fields. Such terms need to be added to the following expressions in order to obtain the complete expressions, as mentioned in Sec. 4.5, We take the notations of Ref. [17] for the component fields.

A hypermultiplet $\left[\mathcal{A}_{i}^{\bar{a}}, \zeta_{\alpha}^{\bar{a}}, \mathcal{F}_{i}^{\bar{a}}\right]\left(i=1,2 ; \bar{a}=1,2, \cdots, 2\left(n_{C}+n_{H}\right)\right)$ is split into two chiral multiplets,

$$
\begin{aligned}
\Phi^{2 a-1} & =\left(1+\frac{1}{2} \mathcal{E}\right)\left\{\mathcal{A}_{2}^{2 a-1}-2 i \theta \zeta^{a-}+\theta^{2} F^{2 a-1}\right\}, \\
\Phi^{2 a} & =\left(1+\frac{1}{2} \mathcal{E}\right)\left\{\mathcal{A}_{2}^{2 a}-2 i \theta \zeta^{a+}+\theta^{2} F^{2 a}\right\},
\end{aligned}
$$

where

$$
F^{\bar{a}} \equiv i \mathcal{F}_{1}^{\bar{a}}+\left(\mathcal{D}_{4} \mathcal{A}\right)_{1}^{\bar{a}}+i g M^{I}\left(t_{I}\right)_{\bar{b}}^{\bar{a}} \mathcal{A}_{1}^{\bar{b}}
$$

and the definition of the covariant derivative $\left(\mathcal{D}_{M} \mathcal{A}\right)_{i}^{\bar{a}}$ is given in Ref. [17]. The 2-component spinor notation of the hyperini, which are the symplectic-Majorana spinor, is defined from the 4-component notation in Ref. [17] as

$$
\zeta^{2 a-1}=\left(\begin{array}{c}
\zeta^{a-} \\
-\bar{\zeta}^{a+}
\end{array}\right), \quad \zeta^{2 a}=\left(\begin{array}{c}
\zeta^{a+} \\
\bar{\zeta}^{a-}
\end{array}\right) .
$$

A $5 \mathrm{D}$ vector multiplet $\left[M^{I}, W_{M}^{I}, \Omega^{I i}, Y^{I r}\right]$ is decomposed into $\mathcal{N}=1$ gauge and chiral superfields as

$$
\begin{aligned}
V^{I}= & -\left(\theta \sigma^{\mu} \bar{\theta}\right)\left(e^{-1}\right)_{\mu}{ }^{\nu} W_{\nu}^{I}+i \theta^{2} \bar{\theta}\left\{2 \bar{\Omega}^{I+}-\left(\bar{\sigma}^{\nu} \sigma^{\mu} \bar{\psi}_{\nu}^{+}\right) W_{\mu}^{I}\right\} \\
& -i \bar{\theta}^{2} \theta\left\{2 \Omega^{I+}-\left(\sigma^{\nu} \bar{\sigma}^{\mu} \psi_{\nu}^{+}\right) W_{\mu}^{I}\right\}+\frac{1}{2} \theta^{2} \bar{\theta}^{2} D^{I}, \\
\Sigma^{I}= & \frac{1}{2}\left(e_{y}{ }^{4} M^{I}-i W_{y}^{I}\right)+\theta\left(2 i e_{y}{ }^{4} \Omega^{I-}+2 \psi_{y}^{-} M^{I}\right) \\
& -\theta^{2}\left\{e_{y}{ }^{4}\left(Y^{I 1}+i Y^{I 2}\right)+i\left(V_{y}^{1}+i V_{y}^{2}\right) M^{I}\right\},
\end{aligned}
$$

where

$$
D^{I} \equiv 2 Y^{I 3}-\left(\mathcal{D}_{4} M\right)^{I}+\left(-2 \bar{\Omega}^{I+} \bar{\sigma}^{\mu} \psi_{\mu}^{+}+\text {h.c. }\right)+\left(2 V^{3 \mu}+2 v^{\mu}{ }_{4}-\frac{1}{2} \epsilon^{\mu \nu \rho \tau} \partial_{\nu} \tilde{e}_{\rho \tau}\right) W_{\mu}^{I} .
$$

The 2-component spinor notation of an $S U(2)_{U}$-Majorana spinor $\psi^{i}=\Omega^{I i}, \psi_{M}^{i}$ is defined from the 4-component notation in Ref. [17] as

$$
\psi^{1}=\left(\begin{array}{c}
\psi^{+} \\
-\bar{\psi}^{-}
\end{array}\right), \quad \psi^{2}=\left(\begin{array}{c}
\psi^{-} \\
\bar{\psi}^{+}
\end{array}\right) .
$$




\section{Invariance of the action}

\section{C.1 Gauge invariance of the action}

Here we check an invariance of the action under the gauge transformation (4.7). The invariance of the hypermultiplet sector is manifest. In the vector multiplet sector, the $d^{4} \theta$-integral part is also manifestly gauge-invariant since it consists of the field strength superfield $\mathcal{V}^{I}$ and the gauge singlet $\tilde{V}_{E}$. Hence the only nontrivial part is the (supersymmetric) Chern-Simons terms,

$$
\mathcal{L}^{\mathrm{CS}} \equiv \int d^{2} \theta W^{\mathrm{CS}}+\text { h.c. }
$$

where $W^{\mathrm{CS}}$ is given by (4.19).

As explained in Sec. 4.2, the dependences of $\mathcal{L}^{\mathrm{CS}}$ on $U^{\mu}$ and $\Psi_{\alpha}\left(\bar{\Psi}_{\dot{\alpha}}\right)$ are not fixed completely only by the $\delta_{\mathrm{sc}}^{(1)}$-invariance, i.e., a constant $a$ in (4.18) remains undetermined. As we will show in the following, the gauge invariance determines its value.

First, $\mathcal{L}^{\mathrm{CS}}$ is split into three parts, i.e., $\left.\mathcal{L}_{0}^{\mathrm{CS}} \equiv \mathcal{L}^{\mathrm{CS}}\right|_{U^{\mu}=\Psi_{\alpha}=0}, U^{\mu}$-dependent part $\Delta_{U} \mathcal{L}^{\mathrm{CS}}$, and $\Psi_{\alpha}\left(\bar{\Psi}_{\dot{\alpha}}\right)$-dependent part $\Delta_{\Psi} \mathcal{L}^{\mathrm{CS}}$. Their explicit forms are

$$
\begin{aligned}
\mathcal{L}_{0}^{\mathrm{CS}}=\int d^{2} \theta \frac{3 C_{I J K}}{2}\left\{-\Sigma^{I} \mathcal{W}_{0}^{J} \mathcal{W}_{0}^{K}+\frac{\bar{D}^{2}}{12}\left(\mathcal{Z}_{0}^{I J \alpha}\right) \mathcal{W}_{0 \alpha}^{K}\right\}+\text { h.c. } \\
\Delta_{U} \mathcal{L}^{\mathrm{CS}}=\int d^{2} \theta \frac{3 C_{I J K}}{2}\left\{-\frac{1}{2} \Sigma^{I} \bar{D}^{2}\left(U^{\mu} \mathcal{W}_{0}^{J} \sigma_{\mu} \overline{\mathcal{W}}_{0}^{K}\right)\right. \\
\left.\quad+\frac{\bar{D}^{2}}{12}\left(\Delta_{U} \mathcal{Z}^{I J \alpha} \mathcal{W}_{0 \alpha}^{K}+\frac{1}{4} U^{\mu} \bar{D}^{2} \mathcal{Z}_{0}^{I J} \sigma_{\mu} \overline{\mathcal{W}}_{0}^{K}\right)\right\}+ \text { h.c. } \\
\Delta_{\Psi} \mathcal{L}^{\mathrm{CS}}=\int d^{2} \theta \frac{C_{I J K}}{8}\left(\Delta_{\Psi} \mathcal{Z}^{I J \alpha} \mathcal{W}_{0 \alpha}^{K}\right)+\text { h.c. }
\end{aligned}
$$

where

$$
\begin{aligned}
\mathcal{Z}_{0}^{I J \alpha} \equiv & X^{I} D_{\alpha} \partial_{y} X^{J}-\partial_{y} X^{I} D^{\alpha} X^{J}, \\
\Delta_{U} \mathcal{Z}^{I J \alpha} \equiv & -\frac{1}{2} \bar{\sigma}_{\mu}^{\dot{\beta} \beta} U^{\mu}\left\{X^{I} D^{\alpha} D_{\beta} \bar{D}_{\dot{\beta}} \partial_{y} X^{J}+D_{\beta} \bar{D}_{\dot{\beta}} X^{I} D^{\alpha} \partial_{y} X^{J}\right. \\
& \left.\quad-\partial_{y} X^{I} D^{\alpha} D_{\beta} \bar{D}_{\dot{\beta}} X^{J}-D_{\beta} \bar{D}_{\dot{\beta}} \partial_{y} X^{I} D^{\alpha} X^{J}\right) \\
& -\frac{1}{2} \bar{\sigma}_{\mu}^{\dot{\beta} \beta} D^{\alpha} U^{\mu}\left(\bar{D}_{\dot{\beta}} X^{I} D_{\beta} \partial_{y} X^{J}-\bar{D}_{\dot{\beta}} \partial_{y} X^{I} D_{\beta} X^{J}\right) \\
& -\frac{a}{4} \bar{\sigma}_{\mu}^{\dot{\alpha} \alpha} \partial_{y} U^{\mu} X^{I} D^{2} \bar{D}_{\dot{\alpha}} X^{J},
\end{aligned}
$$




$$
\begin{aligned}
\Delta_{\Psi} \mathcal{Z}^{I J \alpha} \equiv & -X^{I} D^{\alpha}\left(\frac{1}{4} \bar{D}^{2} \Psi^{\beta} D_{\beta}+\frac{1}{2} \bar{D}^{\dot{\beta}} \Psi^{\beta} \bar{D}_{\dot{\beta}} D_{\beta}+\text { h.c. }\right) X^{J} \\
& +\left(\frac{1}{4} \bar{D}^{2} \Psi^{\beta} D_{\beta}+\frac{1}{2} \bar{D}^{\dot{\beta}} \Psi^{\beta} \bar{D}_{\dot{\beta}} D_{\beta}+\text { h.c. }\right) X^{I} D^{\alpha} X^{J} \\
& -\frac{a}{4}\left(\bar{D}^{\dot{\alpha}} \Psi^{\alpha}-D^{\alpha} \bar{\Psi}^{\dot{\alpha}}\right) X^{I} D^{2} \bar{D}_{\dot{\alpha}} X^{J} .
\end{aligned}
$$

The gauge transformation of $\mathcal{L}_{\mathrm{CS} 0}$ is calculated as

$$
\delta_{\text {gauge }} \mathcal{L}_{0}^{\mathrm{CS}}=-\int d^{2} \theta \frac{3 C_{I J K}}{2}\left\{\frac{1}{2} \partial_{y}\left(\Lambda^{I} \mathcal{W}_{0}^{J} \mathcal{W}_{0}^{K}\right)-\mathcal{O}_{\Psi}^{(1)} \Lambda^{I} \mathcal{W}_{0}^{J} \mathcal{W}_{0}^{K}\right\}+\text { h.c. }
$$

where

$$
\mathcal{O}_{\Psi}^{(1)} \equiv \frac{1}{4} \bar{D}^{2} \Psi^{\beta} D_{\beta}+\frac{1}{2} \bar{D}^{\dot{\beta}} \Psi^{\beta} \bar{D}_{\dot{\beta}} D_{\beta}+\text { h.c.. }
$$

We have used the partial integrals and $D^{\alpha} \mathcal{W}_{0 \alpha}^{K}=\bar{D}_{\dot{\alpha}} \overline{\mathcal{W}}_{0}^{K \dot{\alpha}}$.

After some calculations, the gauge transformation of $\Delta_{U} \mathcal{L}^{\mathrm{CS}}$ is obtained as

$$
\begin{array}{r}
\delta_{\text {gauge }} \Delta_{U} \mathcal{L}^{\mathrm{CS}}=-\int d^{4} \theta \frac{C_{I J K}}{2}\left[U^{\mu} \partial_{y}\left\{\left(\Lambda^{I}+\bar{\Lambda}^{I}\right) \overline{\mathcal{W}}_{0}^{J} \bar{\sigma}_{\mu} \mathcal{W}_{0}^{K}\right\}\right. \\
\left.+a \partial_{y} U^{\mu}\left(\Lambda^{I}+\bar{\Lambda}^{I}\right) \overline{\mathcal{W}}_{0}^{J} \bar{\sigma}_{\mu} \mathcal{W}_{0}^{K}+\text { h.c. }\right] .
\end{array}
$$

Here we have used the relation $-\frac{1}{4} \bar{D}^{2}=d^{2} \bar{\theta}$ up to the total derivative, and the following identity that holds for arbitrary spinors $\chi_{\alpha}, \psi_{\alpha}, \lambda_{\alpha}$.

$$
\psi_{\beta} \chi^{\beta} \lambda^{\alpha}-\psi^{\alpha} \chi^{\beta} \lambda_{\beta}-\psi_{\beta} \chi^{\alpha} \lambda^{\beta}=0
$$

Finally we consider the gauge transformation of $\Delta_{\Psi} \mathcal{L}^{\mathrm{CS}}$.

$$
\begin{aligned}
\delta_{\text {gauge }} \Delta_{\Psi} \mathcal{L}^{\mathrm{CS}} & =-\int d^{4} \theta\left\{\frac{C_{I J K}}{2} \delta_{\text {gauge }} \Delta_{\Psi} \mathcal{Z}^{I J \alpha} \mathcal{W}_{0 \alpha}^{K}+\text { h.c. }\right\} \\
& =\int d^{4} \theta C_{I J K}\left\{\Lambda^{I}\left(\mathcal{O}_{\Psi}^{(2)}\right)_{\beta}^{\alpha} D^{\beta} X^{J}-\mathcal{O}_{\Psi}^{(1)} \Lambda^{I} D^{\alpha} X^{J}\right\} \mathcal{W}_{0 \alpha}^{K}+\text { h.c. }
\end{aligned}
$$

where

$$
\begin{aligned}
\left(\mathcal{O}_{\Psi}^{(2)}\right)_{\beta}^{\alpha}= & \delta_{\beta}^{\alpha}\left(\frac{1}{4} \bar{D}^{2} \Psi^{\gamma} D_{\gamma}+i \sigma_{\gamma \dot{\gamma}}^{\mu} \bar{D}^{\dot{\gamma}} \Psi^{\gamma} \partial_{\mu}\right) \\
& -\left(\frac{1}{4} D^{\alpha} \bar{D}^{2} \Psi_{\beta}+\frac{1}{2} D^{\alpha} \bar{D}^{\dot{\beta}} \Psi_{\beta} \bar{D}_{\dot{\beta}}\right)
\end{aligned}
$$

We have used (4.17) in deriving (C.9). By using (C.8), we can show that

$$
C_{I J K} \mathcal{O}_{\Psi}^{(1)} \Lambda^{I} D^{\alpha} X^{J} \mathcal{W}_{0 \alpha}^{K}=-2 C_{I J K} \Lambda^{I}\left(\mathcal{O}_{\Psi}^{(2)}\right)_{\beta}^{\alpha} D^{\beta} X^{J} \mathcal{W}_{0 \alpha}^{K}
$$


up to total derivatives. Thus, we obtain

$$
\begin{aligned}
\delta_{\text {gauge }} \Delta_{\Psi} \mathcal{L}^{\mathrm{CS}} & =-\int d^{4} \theta \frac{3 C_{I J K}}{2} \mathcal{O}_{\Psi}^{(1)} \Lambda^{I} D^{\alpha} X^{J} \mathcal{W}_{0 \alpha}^{K}+\text { h.c. } \\
& =-\int d^{2} \theta \frac{3 C_{I J K}}{2} \mathcal{O}_{\Psi}^{(1)} \Lambda^{I} \mathcal{W}_{0}^{J} \mathcal{W}_{0}^{K}+\text { h.c.. }
\end{aligned}
$$

Summing up (‥5), (․7) and (․12), we find that $\delta_{\text {gauge }} \mathcal{L}^{\mathrm{CS}}$ becomes the following total derivative when $a=1$.

$$
\begin{aligned}
\delta_{\text {gauge }} \mathcal{L}^{\mathrm{CS}} & =-\int d^{2} \theta \frac{C_{I J K}}{2} \partial_{y}\left\{\Lambda^{I} \mathcal{W}_{0}^{J} \mathcal{W}_{0}^{K}-\frac{1}{2} \bar{D}^{2}\left(U^{\mu} \Lambda^{I} \overline{\mathcal{W}}_{0}^{J} \bar{\sigma}_{\mu} \mathcal{W}_{0}^{K}\right)\right\}+\text { h.c. } \\
& =-\int d^{2} \theta \frac{C_{I J K}}{2} \partial_{y}\left(\Lambda^{I} \mathcal{W}^{J} \mathcal{W}^{K}\right)+\text { h.c.. }
\end{aligned}
$$

Therefore, the gauge invariance of the action determines the value of $a$.

\section{C.2 $\delta_{\mathrm{sc}}^{(2)}$-invariance of the action}

Here we show the invariance of the action under the $\delta_{\mathrm{sc}}^{(2)}$-transformation in (4.30) at the zeroth order in the gravitational superfields. First we consider the hypermultiplet sector. From the definition of $\mathcal{U}$ in (4.32), $\mathcal{U}\left(\Phi^{\bar{a}}\right)$ transforms as

$$
\begin{aligned}
\delta_{\mathrm{sc}}^{(2)} \mathcal{U}\left(\Phi_{\text {odd }}\right) & =\frac{1}{2}(Y+\bar{Y}) \partial_{y} \Phi_{\text {odd }}-\frac{i}{4} \bar{D}^{2}\left\{\tilde{N}\left(e^{-V}\right)^{t} \bar{\Phi}_{\text {even }}\right\}+i \tilde{N} \partial_{y} \Phi_{\text {odd }} \\
\delta_{\text {sc }}^{(2)} \mathcal{U}\left(\Phi_{\text {even }}\right) & =\frac{1}{2}(Y+\bar{Y}) \partial_{y} \Phi_{\text {even }}+\frac{i}{4} \bar{D}^{2}\left\{\tilde{N} e^{V} \bar{\Phi}_{\text {odd }}\right\}+i \tilde{N} \partial_{y} \Phi_{\text {even }} .
\end{aligned}
$$

Then, $\Omega^{\text {hyper }}$ and $W^{\text {hyper }}$ in (4.20) transform as

$$
\begin{aligned}
& \delta_{\mathrm{sc}}^{(2)} \Omega^{\text {hyper }}=-\frac{1}{2} \partial_{y}\left[(Y+\bar{Y})\left\{\Phi_{\text {odd }}^{\dagger} \tilde{d}\left(e^{V}\right)^{t} \Phi_{\text {odd }}+\Phi_{\text {even }}^{\dagger} \tilde{d} e^{-V} \Phi_{\text {even }}\right\}\right] \\
& -\left[\frac{i}{4} \tilde{N}\left(D^{2} \Phi_{\text {odd }}^{t} \tilde{d} \Phi_{\text {even }}-\Phi_{\text {odd }}^{t} \tilde{d} D^{2} \Phi_{\text {even }}\right)-\frac{i}{2} D^{\alpha} \tilde{N} \Phi_{\text {odd }}^{t} \tilde{d} D_{\alpha} e^{V} e^{-V} \Phi_{\text {even }}\right. \\
& +i \tilde{N}\left\{\Phi_{\text {odd }}^{\dagger} \tilde{d}\left(e^{V}\right)^{t} \partial_{y} \Phi_{\text {odd }}+\Phi_{\text {even }}^{\dagger} \tilde{d} e^{-V} \partial_{y} \Phi_{\text {even }}\right\} \\
& \left.+i \tilde{N}\left\{\Phi_{\text {odd }}^{\dagger} \tilde{d}\left(\Sigma e^{V}\right)^{t} \Phi_{\text {odd }}-\Phi_{\text {even }}^{\dagger} \tilde{d}\left(e^{-V} \Sigma\right) \Phi_{\text {even }}\right\}+\text { h.c. }\right], \\
& \delta_{\mathrm{sc}}^{(2)} W^{\text {hyper }}=\partial_{y}\left[Y\left\{\Phi_{\text {odd }}^{t} \tilde{d}\left(\partial_{y}-\Sigma\right) \Phi_{\text {even }}-\Phi_{\text {even }}^{t} \tilde{d}\left(\partial_{y}+\Sigma^{t}\right) \Phi_{\text {odd }}\right\}\right] \\
& -\frac{i}{4} \bar{D}^{2}\left[2 \tilde{N}\left\{\Phi_{\text {odd }}^{\dagger} \tilde{d}\left(e^{V}\right)^{t} \partial_{y} \Phi_{\text {odd }}+\Phi_{\text {even }} \tilde{d} e^{-V} \partial_{y} \Phi_{\text {even }}\right\}\right. \\
& +2 \tilde{N}\left\{\Phi_{\text {odd }}^{\dagger} \tilde{d}\left(\Sigma e^{V}\right)^{t} \Phi_{\text {odd }}-\Phi_{\text {even }}^{\dagger} \tilde{d} e^{-V} \Sigma \Phi_{\text {even }}\right\} \\
& \left.-\frac{1}{2}\left(\Phi_{\text {odd }}^{t} \tilde{d} D^{2} \Phi_{\text {even }}-\Phi_{\text {even }}^{t} \tilde{d} D^{2} \Phi_{\text {odd }}\right)-D^{\alpha} \tilde{N} \Phi_{\text {odd }}^{t} \tilde{d} D_{\alpha} e^{V} e^{-V} \Phi_{\text {even }}\right]
\end{aligned}
$$


where we have dropped total derivatives for $x^{\mu}$ and $\theta_{\alpha}\left(\bar{\theta}_{\dot{\alpha}}\right)$. Thus, we can see that

$$
\delta_{\mathrm{sc}}^{(2)}\left[2 \int d^{4} \theta \Omega^{\text {hyper }}+\left\{\int d^{2} \theta W^{\text {hyper }}+\text { h.c. }\right\}\right]=0,
$$

up to total derivatives, by using $-\frac{1}{4} \bar{D}^{2}=d^{2} \bar{\theta}$. Note that the cancellation occurs between the $\delta_{\mathrm{sc}}^{(2)}$-variations of the $d^{4} \theta$-integral and the $d^{2} \theta$-integral.

Next we consider the vector multiplet sector. For simplicity, we assume that the gauge group is Abelian. From (4.30) and (4.36), we see that

$$
\delta_{\mathrm{sc}}^{(2)}\left(\frac{\mathcal{V}^{I}}{V_{E}}\right)=\frac{1}{2}(Y+\bar{Y}) \partial_{y} \mathcal{V}^{I}-\left\{\frac{i}{2} D^{\alpha}\left(N \mathcal{W}_{\alpha}^{I}\right)+\text { h.c. }\right\} .
$$

The ratio $\mathcal{V}^{I} / V_{E}$ is identified with an $\mathcal{N}=1$ real general multiplet (4.19) in Ref. [17]. The first term in (C.17) corresponds to (4.15) of Ref.[17]. Then we obtain the transformation law of $\Omega^{\text {vector }}$ defined in (4.12) as

$$
\delta_{\text {sc }}^{(2)} \Omega^{\text {vector }}=\partial_{y}\left\{\frac{1}{2}(Y+\bar{Y}) \Omega^{\text {vector }}\right\}-\left\{\frac{3}{8} C_{I J K} \mathcal{V}^{I} \mathcal{V}^{J} D^{\alpha} Y \mathcal{W}_{\alpha}^{K}+\text { h.c. }\right\}
$$

Since it is cumbersome to show the invariance of the whole action in this sector, here we focus on the quadratic terms in $\Sigma^{I}$ in the $\delta_{\mathrm{sc}}^{(2)}$-variation to illustrate the cancellations. Then, $W^{\mathrm{CS}}$ defined in (4.19) transforms as

$$
\begin{aligned}
\delta_{\mathrm{sc}}^{(2)}\left\{\int d^{2} \theta W^{\mathrm{CS}}+\text { h.c. }\right\} & =\int d^{2} \theta \frac{3 C_{I J K}}{2}\left(-2 \Sigma^{I} \delta_{\mathrm{sc}}^{(2)} \mathcal{W}^{J} \mathcal{W}^{K}+\cdots\right)+\text { h.c. } \\
& =-\int d^{2} \theta \frac{3 C_{I J K}}{8} \bar{D}^{2}\left[\Sigma^{I} D^{\alpha}\left\{(Y+\bar{Y}) \mathcal{V}^{J}\right\} \mathcal{W}_{\alpha}^{K}+\cdots\right]+\text { h.c. } \\
& =\int d^{4} \theta \frac{3 C_{I J K}}{4}\left[D^{\alpha} Y\left(\Sigma^{I}+\bar{\Sigma}^{I}\right)\left(\Sigma^{J}+\bar{\Sigma}^{J}\right) \mathcal{W}_{\alpha}^{K}+\cdots+\text { h.c. }\right]
\end{aligned}
$$

where the ellipses denote terms up to linear in $\Sigma^{I}$ or $\bar{\Sigma}^{I}$. We have dropped total derivatives, and used $-\frac{1}{4} \bar{D}^{2}=d^{2} \bar{\theta}$ and the fact that $D^{\alpha} \mathcal{W}_{\alpha}^{I}$ is real at the zeroth order in the gravitational superfields. Therefore, we can see the cancellation between the $\delta_{\mathrm{sc}}^{(2)}$-variation of the $d^{4} \theta$ integral and the $d^{2} \theta$-integral,

$$
\delta_{\mathrm{sc}}^{(2)}\left[2 \int d^{4} \theta \Omega^{\text {vector }}+\left\{\int d^{2} \theta W^{\mathrm{CS}}+\text { h.c. }\right\}\right]=0,
$$

up to total derivatives.

Summing up (C.16) and (C.20), we obtain (4.37). 


\section{References}

[1] J. M. Maldacena, Adv. Theor. Math. Phys. 2 (1998) 231 [Int. J. Theor. Phys. 38 (1999) 1113] hep-th/9711200.

[2] P. Horava and E. Witten, Nucl. Phys. B 460 (1996) 506 [hep-th/9510209]; Nucl. Phys. B 475 (1996) 94 hep-th/9603142].

[3] A. Lukas, B. A. Ovrut, K. S. Stelle and D. Waldram, Phys. Rev. D 59 (1999) 086001 [hep-th/9803235]; Nucl. Phys. B 552 (1999) 246 [hep-th/9806051].

[4] L. Randall and R. Sundrum, Phys. Rev. Lett. 83 (1999) 3370 hep-ph/9905221].

[5] T. Gherghetta and A. Pomarol, Nucl. Phys. B 586 (2000) 141 [hep-ph/0003129].

[6] A. Falkowski, Z. Lalak and S. Pokorski, Phys. Lett. B 491 (2000) 172 [hep-th/0004093].

[7] R. Altendorfer, J. Bagger and D. Nemeschansky, Phys. Rev. D 63 (2001) 125025 hep-th/0003117].

[8] M. Gunaydin, G. Sierra and P. K. Townsend, Nucl. Phys. B 242 (1984) 244; Nucl. Phys. B 253 (1985) 573.

[9] M. Gunaydin and M. Zagermann, Nucl. Phys. B 572 (2000) 131 hep-th/9912027.

[10] A. Ceresole and G. Dall'Agata, Nucl. Phys. B 585, 143 (2000) hep-th/0004111.

[11] E. Bergshoeff, S. Cucu, T. De Wit, J. Gheerardyn, R. Halbersma, S. Vandoren and A. Van Proeyen, JHEP 0210 (2002) 045 [hep-th/0205230].

[12] E. Bergshoeff, S. Cucu, T. de Wit, J. Gheerardyn, S. Vandoren and A. Van Proeyen, Class. Quant. Grav. 21 (2004) 3015 [Class. Quant. Grav. 23 (2006) 7149] hep-th/0403045.

[13] M. Zucker, Nucl. Phys. B 570 (2000) 267 hep-th/9907082.

[14] T. Kugo and K. Ohashi, Prog. Theor. Phys. 105 (2001) 323 hep-ph/0010288.

[15] T. Fujita and K. Ohashi, Prog. Theor. Phys. 106 (2001) 221 [hep-th/0104130]. 
[16] T. Fujita, T. Kugo and K. Ohashi, Prog. Theor. Phys. 106 (2001) 671 hep-th/0106051.

[17] T. Kugo and K. Ohashi, Prog. Theor. Phys. 108 (2002) 203 hep-th/0203276.

[18] T. Kugo and K. Ohashi, Prog. Theor. Phys. 108 (2003) 1143 hep-th/0208082.

[19] S. M. Kuzenko and G. Tartaglino-Mazzucchelli, Phys. Lett. B 661 (2008) 42 arXiv:0710.3440 [hep-th]]; JHEP 0802 (2008) 004 [arXiv:0712.3102 [hep-th]]; JHEP 0804 (2008) 032 [arXiv:0802.3953 [hep-th]].

[20] W. D. Linch, III and G. Tartaglino-Mazzucchelli, arXiv:1204.4195 [hep-th].

[21] N. Arkani-Hamed, T. Gregoire and J. G. Wacker, JHEP 0203 (2002) 055 hep-th/0101233.

[22] D. Marti and A. Pomarol, Phys. Rev. D 64 (2001) 105025 [hep-th/0106256].

[23] A. Hebecker, Nucl. Phys. B 632 (2002) 101 [hep-ph/0112230].

[24] S. M. Kuzenko and W. D. Linch, III, JHEP 0602 (2006) 038 hep-th/0507176.

[25] W. D. I. Linch, M. A. Luty and J. Phillips, Phys. Rev. D 68 (2003) 025008 arXiv:hep-th/0209060.

[26] H. Abe and Y. Sakamura, JHEP 0410 (2004) 013 [hep-th/0408224].

[27] F. Paccetti Correia, M. G. Schmidt, Z. Tavartkiladze and, Nucl. Phys. B 709 (2005) 141 [hep-th/0408138].

[28] H. Abe and Y. Sakamura, Phys. Rev. D 71 (2005) 105010 hep-th/0501183; Phys. Rev. D 73 (2006) 125013 [hep-th/0511208].

[29] F. Paccetti Correia, M. G. Schmidt, Z. Tavartkiladze and, Nucl. Phys. B 751 (2006) 222 hep-th/0602173.

[30] H. Abe and Y. Sakamura, Phys. Rev. D 75 (2007) 025018 hep-th/0610234.

[31] H. Abe and Y. Sakamura, Phys. Rev. D 79 (2009) 045005 [arXiv:0807.3725 [hep-th]].

[32] H. Abe, H. Otsuka, Y. Sakamura and Y. Yamada, arXiv:1111.3721 [hep-ph]. 
[33] Y. Sakamura, JHEP 1112 (2011) 008 [arXiv:1107.4247 [hep-th]].

[34] T. Kugo and S. Uehara, Nucl. Phys. B 226 (1983) 49.

[35] W. Siegel and S. J. Gates, Jr., Nucl. Phys. B 147 (1979) 77.

[36] J. Wess and J. Bagger, Princeton, USA: Univ. Pr. (1992) $259 p$

[37] I. L. Buchbinder and S. M. Kuzenko, Bristol, UK: IOP (1995) 640 p.

[38] A. Hebecker, J. March-Russell and R. Ziegler, JHEP 0908 (2009) 064 arXiv:0801.4101 [hep-ph]].

[39] A. Falkowski, Z. Lalak and S. Pokorski, Phys. Lett. B 509 (2001) 337 [hep-th/0009167].

[40] H. Abe and Y. Sakamura, Nucl. Phys. B 796 (2008) 224 [arXiv:0709.3791 [hep-th]].

[41] I. L. Buchbinder, S. J. Gates, Jr., H. -S. Goh, W. D. Linch, III, M. A. Luty, S. -P. Ng and J. Phillips, Phys. Rev. D 70 (2004) 025008 [hep-th/0305169].

[42] T. Gregoire, R. Rattazzi, C. A. Scrucca, A. Strumia and E. Trincherini, Nucl. Phys. B 720 (2005) 3 [hep-th/0411216].

[43] M. Fabinger and P. Horava, Nucl. Phys. B 580 (2000) 243 hep-th/0002073.

[44] J. Garriga, O. Pujolas and T. Tanaka, Nucl. Phys. B 605 (2001) 192 hep-th/0004109].

[45] H. Abe, T. Kobayashi, H. Ohki and K. Sumita, arXiv:1204.5327 [hep-th]. 